\title{
Blaschke-type conditions on unbounded domains, generalized convexity, and applications in perturbation theory
}

\author{
Sergey Favorov and Leonid Golinskii
}

\begin{abstract}
We introduce a new geometric characteristic of compact sets in the plane called $r$-convexity, which fits nicely into the concept of generalized convexity and extends standard convexity in an essential way. We obtain a Blaschke-type condition for the Riesz measures of certain subharmonic functions on unbounded domains with $r$-convex complements, having growth governed by the distance to the boundary. The result is applied to the study of the convergence of the discrete spectrum for the Schatten-von Neumann perturbations of bounded linear operators in Hilbert space.
\end{abstract}

\section{Introduction}

In 1915 Blaschke [3] proved his celebrated result concerning zero sets of bounded analytic functions in the unit disk, which is a gem of function theory. A vast literature with various refinements and far reaching extensions of the Blaschke condition has appeared since; see [9], [18], [22], [35], and [39], and references therein.

We focus on a series of recent papers ([4], [12], [13], [17]), where the authors study the zero sets of functions analytic in the unit disk, which grow in the direction of a prescribed subset of the unit circle. The result in [12] for analytic functions is as follows.

Theorem A. Let $E \subset \partial \mathbb{D}$ be a closed subset of the unit circle, and let $f$ be an analytic function in the unit disk $\mathbb{D}$ with zero set $Z_{f}=\left\{z_{n}\right\}$ (each zero $z_{n}$ enters with its multiplicity) so that $|f(0)|=1$, and

$$
\log |f(z)| \leq \frac{K_{f}}{\operatorname{dist}^{q}(z, E)}, \quad z \in \mathbb{D}, \quad q>0,
$$

Mathematics Subject Classification (2010): Primary 31A05; Secondary 31A10, 47A55, 47A10, 47B10.

Keywords: Subharmonic functions, Riesz measure, Riesz decomposition theorem, Green's function, Schatten-von Neumann operators. 
where $\operatorname{dist}\left(E_{1}, E_{2}\right)$ is the Euclidian distance between closed sets $E_{1}$ and $E_{2}$. Then for each $\varepsilon>0$,

$$
\sum_{n}\left(1-\left|z_{n}\right|\right) \operatorname{dist}^{p}\left(z_{n}, E\right) \leq C(q, E, \varepsilon) K_{f}, \quad p=\max (q+\kappa(E)-1+\varepsilon, 0),
$$

where $\kappa(E)$ is the upper Minkowski dimension of $E$.

This result can be used in perturbation theory of bounded linear operators, although the situation there is somewhat different. The point is that the basic objects, the resolvent and the perturbation determinant, are analytic functions on the resolvent set (including infinity) of the corresponding operator, which is an unbounded open set of the plane with compact complement $E$, the spectrum of the operator. To handle this problem, attempts were made to transfer the problem to the unit disk, using conformal mapping [8], [21], or the uniformization theorem [17], apply Theorem A and then return to the initial setting by means of certain distortion results. Such attempts were by and large successful only in the cases when $E$ is a single segment [8], [21] or a finite union of disjoint segments [17], and it is absolutely unclear whether it is possible to make such an argument work for an arbitrary compact subset of the line.

The reasoning in [12] reveals the potential theoretic character of the problem, so the natural setting is subharmonic functions $v$ and their Riesz measures (generalized Laplacians) $\mu=1 /(2 \pi) \Delta v$ rather than analytic functions and their zero sets. In the case $v=\log |f|$ with an analytic function $f$, the Riesz measure is a discrete and integer-valued measure supported on $Z_{f}$, and $\mu\{z\}$ equals the multiplicity of the zero at $z$.

In this paper we develop a straightforward approach to the study of subharmonic functions on unbounded domains with growth governed by the distance to the boundary. Let $E$ be a compact set in the complex plane $\mathbb{C}$, which does not split the plane (its complement

$$
\Omega=\overline{\mathbb{C}} \backslash E
$$

in the extended plane $\overline{\mathbb{C}}$ is a domain, that is, a connected open set $\overline{\mathbb{C}}$ ). Consider a class of subharmonic functions on $\Omega$ subject to the growth and normalization conditions

$$
v(z) \leq \psi(d(z)), \quad v(\infty)=0
$$

where

$$
d(z):=\operatorname{dist}(z, E)
$$

is the distance from $z$ to $E$ and $\psi$ is a positive and monotone decreasing function on $\mathbb{R}_{+}=[0, \infty)$.

In the study of the Riesz measures of such functions one is faced with at least two obstacles. First, the set $E$ may be so small (polar or finite), that to apply standard techniques from potential theory we must work in the "outer neighborhood"

$$
\Omega_{t}:=\{z \in \mathbb{C}: d(z)>t\}, \quad t>0, \quad \Omega=\Omega_{0} .
$$


Its boundary $\partial \Omega_{t}=\{z: d(z)=t\}$ is nonpolar, since it splits the plane (see Theorem 3.6.3 in [33]), so the Green's function $G_{t}$ for $\Omega_{t}$ exists and is unique, whenever $\Omega_{t}$ is a domain.

Second, it is not hard to construct a compact set $E$ so that $\Omega$ is a domain, but $\Omega_{t}$ is not for $t>0$. To cope with this problem we introduce a new geometric characteristic, $r$-convexity, which fits nicely in the context of generalized convexity; see [7], [37]. It can be defined in an arbitrary metric space. No linear structure is needed. Precisely, it is well known that a closed set in $\mathbb{C}$ is convex if and only if it is the intersection of all closed half-planes containing it. For an arbitrary closed set $E$ this intersection agrees with the convex hull of $E$. As usual, we denote by

$$
\begin{aligned}
B(x, r) & =\{z:|z-x|<r\}, \quad B^{c}(x, r)=\{z:|z-x| \geq r\}, \\
\partial B(x, r) & =\{z:|z-x|=r\}
\end{aligned}
$$

an open disk of radius $r$ centered at $x$, its complement, and its boundary, respectively. By replacing half-planes with exteriors of open disks $B^{c}$, we come to the following extension of the usual notion of convexity. We start with the obvious inclusion

$$
E \subset \operatorname{conv}_{r}(E):=\bigcap\left\{B^{c}(z, r): E \subset B^{c}(z, r)\right\}, \quad r>0 .
$$

Definition 1.1. We say that a closed set $E$ is $r$-convex, if $E=\operatorname{conv}_{r}(E)$. The set $\operatorname{conv}_{r}(E)$ is called the $r$-convex hull of $E$.

In other words, $E$ is $r$-convex if

$$
\mathbb{C} \backslash E=\bigcup\{B(z, r): B(z, r) \subset \mathbb{C} \backslash E\},
$$

that is, the complement of $E$ can be covered by open disks of a fixed radius $r>0$ which belong to this complement. As for the usual notion of convexity, the intersection of any family of $r$-convex sets is $r$-convex. On the other hand, in contrast to the usual convexity, a finite union of disjoint $r$-convex sets is $r^{\prime}$-convex for some $r^{\prime} \leq r$. It is also clear that $E_{1} \subset E_{2}$ implies $\operatorname{conv}_{r}\left(E_{1}\right) \subset \operatorname{conv}_{r}\left(E_{2}\right)$.

It follows from (1.2), that each $r$-convex set is also $r^{\prime}$-convex for any $r^{\prime}<r$. So it is natural to consider the number $r_{0}(E):=\sup \left\{r: E=\operatorname{conv}_{r}(E)\right\}$, called the radius of convexity of $E$. For instance, each closed convex set $E$ is $r$-convex with $r_{0}(E)=\infty$, and it is easy to see that the same holds for each closed subset of a line. Indeed, any open subset of a line complementary to a closed set can be covered with a disk of arbitrarily large radius. A complete characterization of compact sets $E$ with $r_{0}(E)=\infty$ is given in Proposition 1 . We show that $r_{0}(E)=R$ for each compact subset of a circle $\partial B(x, R)$, which contains more than two points (see Proposition 2.2). The sets with "interior angles", like $\{z \in \overline{\mathbb{D}}: \pi / 4 \leq \arg z \leq$ $7 \pi / 4\}$, are not $r$-convex for any $r>0$.

It turns out (see Theorem 2.9) that if an $r$-convex compact set $E$ does not split the plane, then there exists $t_{0}=t_{0}(E)>0$ such that $\Omega_{t}$ is a domain for all $0 \leq t \leq t_{0}$. So for such $t$ the Green's function $G_{t}$ for $\Omega_{t}$ exists and is unique. A key potential theoretic result (Lemma 3.3) provides the lower bound

$$
G_{t}(z, \infty) \geq C(E) \min \{1, d(z)\}, \quad z \in \Omega_{3 t}, \quad 0<t \leq t_{0},
$$


for the Green's function with its pole at infinity. When $E$ is a finite set, the result can be improved to $G_{t}(z, \infty) \geq C>0, z \in \Omega_{k t}$, with some $k=k(E)>1$. For various estimates of the Green's functions and harmonic measures see, for instance, [29], [14], and [6].

Here is the main result of the paper.

Theorem 1.2. Let $E$ be an $r$-convex compact set with connected complement $\Omega=$ $\overline{\mathbb{C}} \backslash E$, and let $v$ be a subharmonic function satisfying ${ }^{1}$

$$
v(z) \leq K_{v} \psi(d(z)), \quad v(\infty)=0 .
$$

Let $\varphi$ be a positive, monotone increasing, and absolutely continuous function on $\mathbb{R}_{+}$, such that $\varphi_{1}(t):=t^{-1} \varphi(t)$ is monotone increasing in some neighborhood of the origin, $\varphi_{1}(0)=0$, and

$$
\int_{0} \varphi_{1}^{\prime}(t) \psi\left(\frac{t}{3}\right) d t+\int^{\infty} \varphi^{\prime}(t) \psi\left(\frac{t}{3}\right) d t<\infty
$$

(the integrals converge near the origin and infinity, respectively). Then there holds the Blaschke-type condition

$$
\int_{\Omega} \varphi(d(\zeta)) \mu(d \zeta) \leq C(E, \psi, \varphi) K_{v}
$$

for the Riesz measure $\mu$ of $v$. If $v$ is bounded from above in $\Omega, v \leq K_{v}$, then

$$
\int_{\Omega} \min \{1, d(\zeta)\} \mu(d \zeta) \leq C(E) K_{v}
$$

Actually, the second statement is just the Blaschke condition for subharmonic functions on unbounded domains, which might be known to experts. Note that if $E$ is a polar set, then $E$ is removable for the class of bounded from above subharmonic functions. Hence in this case $v$ is constant.

Remark 1.3. Let $E$ be an $r$-convex compact set, and let $\widetilde{\Omega}$ be its outer domain, that is, the unbounded component of $\Omega$. Then the set $\operatorname{Pc}(E)=\overline{\mathbb{C}} \backslash \widetilde{\Omega}$, known as the polynomial convex hull of $E$, is $r$-convex, and $d(z)=\operatorname{dist}(z, \operatorname{Pc}(E))$ for $z \in \tilde{\Omega}$. Given a subharmonic function $v$ satisfying (1.3), its restriction $\tilde{v}$ to $\widetilde{\Omega}$ satisfies the conditions of Theorem 1.2, so the Blaschke-type condition (1.5) holds with $\Omega$ replaced with $\widetilde{\Omega}$.

If $\psi(x)=x^{-q}, q>0$, which is typical in the application of Theorem 1.2, we can take

$$
\varphi(x)=x^{q+1 / 2}(\min \{x, 1 / x\})^{\varepsilon+1 / 2}=\left\{\begin{array}{ll}
x^{q+1+\varepsilon}, & x \leq 1, \\
x^{q-\varepsilon}, & x>1,
\end{array} \quad 0<\varepsilon<q .\right.
$$

\footnotetext{
${ }^{1}$ We single out the constant $K_{v}$ on purpose, in view of applications to perturbation theory in Section 5 .
} 
A special case of Theorem 1.2 with $v=\log |f|, f$ an analytic function, occurs in perturbation theory in the study of discrete spectra for the Schatten-von Neumann perturbations of certain bounded linear operators. Given a bounded linear operator $A_{0}$ on the Hilbert space $\mathcal{H}$, and a compact operator $B$, the fundamental theorem of Weyl states that the essential spectra of $A_{0}$ and $A=A_{0}+B$ agree, so the discrete spectrum of $A$ (the set of isolated eigenvalues of finite algebraic multiplicity) can accumulate only at the joint essential spectrum. We want to gather some information on the rate of accumulation under the stronger assumption that $B$ belongs to some Schatten-von Neumann operator ideal $\mathcal{S}_{q}, 1 \leq q<\infty$, that is, if $\|B\|_{\mathcal{S}_{q}}^{q}:=\sum_{n} s_{n}^{q}(B)<\infty, s_{n}(B)$ are the singular values of $B$.

A number of results of the form

$$
\sum_{\lambda \in \sigma_{d}(A)} d^{p}(\lambda) \leq C\|B\|_{\mathcal{S}_{q}}^{q}, \quad d(\lambda):=\operatorname{dist}\left(\lambda, \sigma\left(A_{0}\right)\right)
$$

with some $p=p(q)$ and $\sigma(T)\left(\sigma_{d}(T)\right)$ the spectrum (discrete spectrum) of an operator $T$, are known. Kato in [23] proved (1.7) for self-adjoint $A_{0}$ and $p=q \geq 1$, $C=1$. Recently Hansmann in [20] obtained the same result for a self-adjoint $A_{0}$ and an arbitrary $B \in \mathcal{S}_{q}$ with $p=q>1$ and the explicit (in a sense) constant $C=C_{q}>1$.

For more general classes of operators (1.7) is known to be true for both $A_{0}$ and $B$ normal with $p=q \geq 2, C=1$, for all three $A_{0}, B$ and $A$ normal with $p=q \geq 1, C=1$, and for $A_{0}$ normal, an arbitrary $B \in \mathcal{S}_{q}$ with $p=q \geq 1$ and $C=1$, under the additional assumption that $\sigma\left(A_{0}\right)$ is a convex set, see [5], [2], and [19], respectively.

We apply Theorem 1.2 for the study of the rate of accumulation of the eigenvalues for an arbitrary $B \in \mathcal{S}_{q}$ and operators $A_{0}$ (in general, nonnormal) with $r$-convex spectrum and growth of the resolvent norm of $A_{0}$ governed by the distance to the spectrum (see the conditions (i)-(iii) in Section 5). The corresponding bound is as follows:

$$
\sum_{\lambda \in \sigma_{d}(A)} \Phi(d(\lambda)) \leq C\|B\|_{\mathcal{S}_{q}}^{q}
$$

where $\Phi$ is a continuous function on $\mathbb{R}_{+}$and $\Phi(0)=0$. This result is illustrated by several examples at the end of the paper.

Although our result on the rate of accumulation does not seem to be optimal, it enables one to extend considerably the class of unperturbed operators with the norm of resolvent growing fast near the spectrum (see, e.g., (5.3) and (5.4)).

We proceed as follows. Section 2 concerns the notion of $r$-convexity and its properties. In Section 3 we obtain a lower bound for the Green's function of an unbounded domain with a compact $r$-convex compliment. Our main result (Theorem 1.2) is proved in Section 4. Applications to perturbation theory are given in Section 5 . 


\section{2. $r$-convexity}

We begin with a characterization of $r$-convex compact sets $E$ with $r_{0}(E)=\infty$.

Proposition 2.1. A compact set $E$ with $r_{0}(E)=\infty$ is either a convex set or a compact subset of a line. In particular, if an $r$-convex compact set $E$ with $r_{0}(E)=\infty$ is connected, then it is convex.

Proof. Assume that $E$ is not convex. Then there is a pair of points $A, B \in E$ so that the open interval $(A, B) \subset \Omega=\overline{\mathbb{C}} \backslash E$. With no loss of generality we set $A=1$ and $B=-1$. Then the vertical interval $[-i \varepsilon, i \varepsilon]$ is contained in $\Omega$ for sufficiently small $\varepsilon$.

We call a disk $B(z, r) \varepsilon$-admissible if $B(z, r) \subset \Omega$ and $i \varepsilon \in B(z, r)$. By assumption there are $\varepsilon$-admissible disks of arbitrarily large radius. It is easy to see that the centers of such disks lie in the sector

$$
\Gamma=\left\{y>\frac{|x|}{\varepsilon}-\frac{1-\varepsilon^{2}}{2 \varepsilon}\right\}
$$

or, otherwise, the center is closer to $A$ or $B$ than to $i \varepsilon$. So there are $\varepsilon$-admissible disks $B(z, r)$ with arbitrarily large $y=\operatorname{Im} z>0$.

Fix $z_{0}=x_{0}+i y_{0}$ with $y_{0}>0$. We show that $z_{0}$ belongs to some $\varepsilon$-admissible disk. Take $0<\varepsilon<y_{0}\left(1+\left|x_{0}\right|\right)^{-1}$ and consider an $\varepsilon$-admissible disk $B(z, r)$ with $z=x+i y \in \Gamma$ and sufficiently large $y>0$. Then

$$
|z-i \varepsilon|^{2}-\left|z-z_{0}\right|^{2}=2 y\left(y_{0}-\varepsilon\right)+2 x x_{0}+\varepsilon^{2}-x_{0}^{2}-y_{0}^{2} .
$$

Since

$$
\varepsilon y+\frac{1-\varepsilon^{2}}{2}>|x|, \quad-2|x|>-2 \varepsilon y-\left(1-\varepsilon^{2}\right), \quad(x, y) \in \Gamma,
$$

we obtain

$$
\begin{aligned}
|z-i \varepsilon|^{2}-\left|z-z_{0}\right|^{2} & \geq 2 y\left(y_{0}-\varepsilon\right)-2|x|\left|x_{0}\right|+\varepsilon^{2}-x_{0}^{2}-y_{0}^{2} \\
& >2 y\left(y_{0}-\varepsilon-\varepsilon\left|x_{0}\right|\right)+\varepsilon^{2}-x_{0}^{2}-y_{0}^{2}>0
\end{aligned}
$$

for sufficiently large $y$ due to the choice of $\varepsilon$. Hence $z_{0} \in B(z, r) \subset \Omega$, as claimed.

The reasoning for $y_{0}<0$ is exactly the same, so once $E$ is not convex, then $E \subset \mathbb{R}$. The proof is complete.

Given an $r$-convex set $E$, it is in general hard to compute its radius of convexity. In some simple instances we can solve this problem.

Proposition 2.2. Let $E=\{a, b, c\}$ be a 3-point set in a general position and let $R=R(a b c)$ be the circumradius of the triangle $\Delta=\Delta(a b c)$. Then $r_{0}(E)=R(a b c)$. Let $E$ be a compact subset of the circle $\partial B(y, \rho)$ such that $|E| \geq 3$. Then $r_{0}(E)=\rho$.

Proof. We recall some facts from elementary planar geometry. A triangle $\Delta(a b c)$ is always viewed as an open planar set. 
1. Given a triangle $\Delta(a b c)$ and $r>R$, there exists exactly one disk $B(x, r)$ such that two vertices (say, $a$ and $b$ ) lie on its boundary, and $c \notin B(x, r)$. We call this disk the $r$-disk and denote it by $(a b)_{r}$. If $r=R$ and $\Delta$ is acute, the $R$-disks $(a b)_{R}$, $(a c)_{R}$, and $(b c)_{R}$ are by definition the reflections of the circumdisk $B_{\Delta}$ through the sides of $\Delta$. If $\Delta$ is not acute, and $c$ is the vertex at the largest angle, then $(a c)_{R}$ and $(b c)_{R}$ are defined as above, and $(a b)_{R}=B_{\Delta}$.

2. If $\Delta$ is acute, then the circles $\partial(a b)_{R}, \partial(a c)_{R}$, and $\partial(b c)_{R}$ meet at one point in $\Delta$, precisely at the orthocenter of $\Delta$ (see, e.g., Problem 5.9 in [31]). If $\Delta$ is not acute, and $c$ is the vertex at the largest angle, the circles $\partial(a b)_{R}, \partial(a c)_{R}$, and $\partial(b c)_{R}$ meet at $c$, and there is a circular triangle with one vertex at $c$, which lies in $\Delta \backslash\left(\overline{(a c)_{R}} \cup \overline{\left.(b c)_{R}\right)}\right.$.

3. For $r \geq R$ let $[a b]_{r}$ be the segment of the disk $(a b)_{r}$ with vertices $a$ and $b$, which intersects $\Delta$. Then for $R \leq r_{1}<r_{2}$ we have $[a b]_{r_{2}} \subset[a b]_{r_{1}}$, and the inclusion is proper.

It is clear that $r_{0}(E) \geq R$, so we wish to show that the complement to $E$ cannot be covered with open disks of radius $r>R$ which lie in this complement. Since $\Delta$ is convex, we can restrict our attention to the points of $\Delta$. Assume that each point $x \in \Delta$ belongs to such disks. Then $x$ belongs to one of the three segments from 3 , so $\Delta \subset\left([a b]_{r} \cup[a c]_{r} \cup[b c]_{r}\right)$. But by 2 and 3 , the latter union can not cover all $\Delta$. This contradiction completes the proof of the first statement.

As far as the second statement goes, the set $E$ is clearly $r$-convex for $r \leq \rho$. For $r>\rho$, as has just been proved, any 3-point set $E_{1}=\{a, b, c\} \subset E$ is not $r$ convex, and $\operatorname{conv}_{r}\left(E_{1}\right)$ contains points from $\Delta(a b c) \subset B(y, \rho)$. Since $\operatorname{conv}_{r}\left(E_{1}\right) \subset$ $\operatorname{conv}_{r}(E)$ for $E_{1} \subset E, E$ cannot be $r$-convex either, as claimed.

Remark 2.3. Given a triangle $\Delta(a b c)$ with circumradius $R$, let $E \subset \partial \Delta$ be a compact set, which contains the vertices $a, b$, and $c$. It follows from the above proof and monotonicity of the $r$-convex hull, that for $r>R$ the intersection of $\operatorname{conv}_{r}(E)$ and $\Delta$ contains a nonempty open set.

To extend the above result, we say that a compact set $E$ has finite global curvature if

$$
r_{g}(E):=\inf \{R(a b c)\}>0,
$$

where the infimum is taken over all possible triangles with vertices in $E$. Clearly, (2.1) holds for finite sets. When $E$ is a Jordan rectifiable curve, the value $r_{g}^{-1}(E)$ is known as the global curvature of $E$; see [34].

Proposition 2.4. Each compact set $E$ with finite global curvature is $r$-convex for some $r>0$, and

$$
r_{0}(E)=r_{g}(E)
$$

Proof. Assume that for some $r>r_{g}(E)$ the set $E$ is $r$-convex. Take a triangle $\Delta\left(a_{0} b_{0} c_{0}\right)$ with $a_{0}, b_{0}, c_{0} \in E$ so that $r>R\left(a_{0} b_{0} c_{0}\right)$. As was shown in the proof of Proposition 2.2, the $r$-convex hull of this triangle (and, moreover, the $r$-convex hull of $E$ itself) contains an open subset of $\Delta\left(a_{0} b_{0} c_{0}\right)$. But it is easily seen from (2.1) that $E$ has empty interior. This contradiction shows that $r_{0}(E) \leq r_{g}(E)$. 
There remains only to show the converse inequality. We show that each point $z \in \mathbb{C} \backslash E$ can be covered with a disk $B \subset \mathbb{C} \backslash E$ of radius at least $r_{g}(E)$. Define

$$
\rho_{z}:=\sup \{r: z \in B(x, r) \subset \mathbb{C} \backslash E\} .
$$

A compactness argument shows that there is a disk $B\left(x_{z}, \rho_{z}\right)$ with

$$
z \in B\left(x_{z}, \rho_{z}\right) \subset \mathbb{C} \backslash E .
$$

If $\partial B\left(x_{z}, \rho_{z}\right) \cap E$ contains at least 3 different points, then $\rho_{z} \geq r_{g}(E)$, as needed. Assume that $\partial B\left(x_{z}, \rho_{z}\right) \cap E=\left\{\zeta_{1}\right\}$, or $\partial B\left(x_{z}, \rho_{z}\right) \cap E=\left\{\zeta_{1}, \zeta_{2}\right\}$, and the points $\zeta_{1}$ and $\zeta_{2}$ do not belong to a diameter of the circle. Then we can shift the disk in an appropriate direction (perpendicular to the interval $\left[\zeta_{1}, \zeta_{2}\right]$ towards the center of the circle), and inflate it a bit to obtain a bigger disk with the same property, which contradicts the maximality $(2.2)$ of $\rho_{z}$.

Hence we can restrict attention to the case where $x_{z}=\rho_{z}, \partial B\left(\rho_{z}, \rho_{z}\right) \cap$ $E=\{a, b\}, a=0$ and $b=2 \rho_{z}$ (after an affine transformation of the plane). Let $G=\left\{z: 0 \leq \operatorname{Re} z \leq 2 \rho_{z}\right\}$.

Assume first that there is a sequence $c_{n}=x_{n}+i y_{n} \in G \cap E$ with $c_{n} \rightarrow a$ or $c_{n} \rightarrow b$ (with no loss of generality let the first relation hold). We want to show that $R\left(a b c_{n}\right) \rightarrow \rho_{z}$. To this end, we apply the explicit formula

$$
R^{-2}\left(z_{1} z_{2} z_{3}\right)=\sum_{\pi} \frac{1}{\left(z_{\pi(1)}-z_{\pi(2)}\right) \overline{\left(z_{\pi(1)}-z_{\pi(3)}\right)}}=\frac{4 \operatorname{Im}^{2}\left(z_{1}-z_{2}\right) \overline{\left(z_{2}-z_{3}\right)}}{\left|\left(z_{1}-z_{2}\right)\left(z_{1}-z_{3}\right)\left(z_{2}-z_{3}\right)\right|^{2}}
$$

for the circumradius $R\left(z_{1} z_{2} z_{3}\right)$, given in [27], where the sum is taken over all permutations of $\{1,2,3\}$. Since $c_{n} \in G \backslash B\left(\rho_{z}, \rho_{z}\right), c_{n} \rightarrow 0$, it is easy to see that $x_{n} / y_{n} \rightarrow 0$ as $n \rightarrow \infty$. It follows from (2.3) that

$$
\begin{aligned}
R^{-2}\left(a b c_{n}\right) & =\frac{16 \rho_{z}^{2} y_{n}^{2}}{4 \rho_{z}^{2}\left|2 \rho_{z}-x_{n}-i y_{n}\right|^{2}\left(x_{n}^{2}+y_{n}^{2}\right)}=\frac{4 y_{n}^{2}}{\left(\left(2 \rho_{z}-x_{n}\right)^{2}+y_{n}^{2}\right)\left(x_{n}^{2}+y_{n}^{2}\right)} \\
& =\frac{4}{\left(\left(2 \rho_{z}-x_{n}\right)^{2}+y_{n}^{2}\right)\left(x_{n}^{2} / y_{n}^{2}+1\right)} \rightarrow \frac{1}{\rho_{z}^{2}},
\end{aligned}
$$

as claimed. Hence $\rho_{z} \geq r_{g}(E)$.

If there exists no such sequence $c_{n}$, the disk can be shifted and inflated, as above, which contradicts maximality of $\rho_{z}$. The proof is complete.

Proposition 2.5. Each $C^{2}$-smooth Jordan curve (arc) has finite global curvature.

Proof. Assume on the contrary, that $r_{g}(\Gamma)=0$ where $\Gamma$ is a $C^{2}$-smooth Jordan curve or arc. Then there is a sequence of triangles $\Delta\left(a_{n} b_{n} c_{n}\right)$ with $a_{n}, b_{n}, c_{n} \in \Gamma$ such that $R\left(a_{n} b_{n} c_{n}\right) \rightarrow 0$ as $n \rightarrow \infty$. By taking subsequences, if needed, we have $a_{n} \rightarrow a \in \Gamma$, and so $b_{n} \rightarrow a$ and $c_{n} \rightarrow a$ as $n \rightarrow \infty$.

On the other hand, we will show that

$$
\lim _{n \rightarrow \infty} R^{-1}\left(a_{n} b_{n} c_{n}\right)=\tau(a)<\infty
$$


where $\tau(a)$ is the curvature of $\Gamma$ at $a$, which will lead to contradiction. Let

$$
\Gamma=\{z(t)=x(t)+i y(t)\}, \quad\left(a_{n} b_{n} c_{n}\right)=\left(z\left(t_{1}\right) z\left(t_{2}\right) z\left(t_{3}\right)\right), \quad a=z(0) .
$$

We apply (2.3) again to obtain

$$
\begin{aligned}
\operatorname{Im}\left(z\left(t_{1}\right)-z\left(t_{2}\right)\right) \overline{\left(z\left(t_{2}\right)-z\left(t_{3}\right)\right)}= & \left(y\left(t_{1}\right)-y\left(t_{2}\right)\right)\left(x\left(t_{2}\right)-x\left(t_{3}\right)\right) \\
& -\left(x\left(t_{1}\right)-x\left(t_{2}\right)\right)\left(y\left(t_{2}\right)-y\left(t_{3}\right)\right),
\end{aligned}
$$

so

$$
\begin{aligned}
\frac{\operatorname{Im}\left(z\left(t_{1}\right)-z\left(t_{2}\right)\right) \overline{\left(z\left(t_{2}\right)-z\left(t_{3}\right)\right)}}{\left(t_{1}-t_{2}\right)\left(t_{2}-t_{3}\right)\left(t_{1}-t_{3}\right)} & =\frac{\left[t_{1} t_{2}\right]_{y}\left[t_{2} t_{3}\right]_{x}-\left[t_{1} t_{2}\right]_{x}\left[t_{2} t_{3}\right]_{y}}{t_{1}-t_{3}} \\
& =\left[t_{1} t_{2} t_{3}\right]_{y}\left[t_{2} t_{3}\right]_{x}-\left[t_{1} t_{2} t_{3}\right]_{x}\left[t_{2} t_{3}\right]_{y}
\end{aligned}
$$

where

$$
\left[t_{i} t_{k}\right]_{f}:=\frac{f\left(t_{i}\right)-f\left(t_{k}\right)}{t_{i}-t_{k}}, \quad\left[t_{1} t_{2} t_{3}\right]_{f}:=\frac{\left[t_{1} t_{2}\right]_{f}-\left[t_{2} t_{3}\right]_{f}}{t_{1}-t_{3}}
$$

are divided differences of the first and second order, respectively. The limit relation

$$
\lim _{t_{i} \rightarrow 0}\left[t_{1} t_{2} t_{3}\right]_{f}=\frac{1}{2} f^{\prime \prime}(0)
$$

provided $f$ is a $C^{2}$-smooth function at the origin, is one of the basic properties of divided differences (see, e.g., [28], page 12). Hence

$$
\lim _{t_{i} \rightarrow 0} \frac{\operatorname{Im}\left(z\left(t_{1}\right)-z\left(t_{2}\right)\right) \overline{\left(z\left(t_{2}\right)-z\left(t_{3}\right)\right)}}{\left(t_{1}-t_{2}\right)\left(t_{2}-t_{3}\right)\left(t_{1}-t_{3}\right)}=\frac{y^{\prime \prime}(0) x^{\prime}(0)-x^{\prime \prime}(0) y^{\prime}(0)}{2},
$$

and finally, by (2.3) and the definition of the curvature,

$$
\lim _{t_{i} \rightarrow 0} R^{-1}\left(z\left(t_{1}\right) z\left(t_{2}\right) z\left(t_{3}\right)\right)=\frac{\left|y^{\prime \prime}(0) x^{\prime}(0)-x^{\prime \prime}(0) y^{\prime}(0)\right|}{\left|z^{\prime}(0)\right|^{3}} .
$$

This is (2.4), as claimed.

Remark 2.6. Proposition 2.5 is a particular case of the much more sophisticated Theorem 1 (iii) in [34], which claims that $\Gamma$ has finite global curvature if and only if its arc length parametrization $\tau(s)$ is $C^{1}$, and $\tau^{\prime}$ satisfies the Lipschitz condition with Lipschitz constant $r_{g}^{-1}(E)$.

Yet another example arises in the theory of elliptic equations in domains with nonsmooth boundaries [1].

Definition 2.7. A planar domain $\Omega$ with boundary $\partial \Omega$ is said to satisfy the uniform ball condition if there is $r>0$ so that for each $x \in \partial \Omega$ there is a ball $B$ of radius $r$ such that

$$
B \subset \Omega, \quad x \in \partial B .
$$

Let $\Gamma$ be a Jordan curve, and let $\mathbb{C} \backslash \Gamma=\Omega_{i} \cup \Omega_{o}$ be the interior and exterior domains of $\Gamma$. We say that $\Gamma$ is a BC-curve if both $\Omega_{i}$ and $\Omega_{o}$ satisfy the uniform ball condition. A Jordan arc $\gamma$ is a BC-arc if there is a BC-curve $\Gamma \supset \gamma$. 
It is easy to see that if $E$ is an $r$-convex compact set then $\Omega=\mathbb{C} \backslash E$ satisfies the uniform ball condition. Indeed, let $x \in \partial \Omega$. There is a sequence of points $z_{n} \in \Omega$ so that $z_{n} \rightarrow x$ as $n \rightarrow \infty$. Take the corresponding sequence of disks $B_{n}$ of radius $r, z_{n} \in B_{n} \subset \Omega$. Then a certain subsequence of $B_{n}$ converges to $B$ in Definition 2.7.

Proposition 2.8. A Jordan curve (arc) is $r$-convex for some $r>0$ if and only if it is a BC-curve (arc).

Proof. Due to the above remark we need to show that each BC-curve (arc) is $r$-convex for some $r>0$. So let $\Gamma$ be a BC-curve, let $z \in \Omega_{i}$, and suppose $d(z)=d(z, \Gamma)<r$. Take $\zeta \in \Gamma$ with $|z-\zeta|=d(z)$ and the "supporting" disks $B_{i}$ and $B_{0}$ of radius $r$ at the point $\zeta$ as in Definition 2.7. Since $B_{i} \subset \Omega_{i}$ and $B_{o} \subset \Omega_{o}$, the disks touch each other at $\zeta$. The disk $B(z, d(z)) \subset \Omega_{i}$ passes through $\zeta$, hence it is necessarily contained in $B_{i}$ and touches $B_{i}$ at $\zeta$. So $z \in B_{i}$, as needed. The argument for $z \in \Omega_{o}$ is the same.

As for the BC arc $\gamma$, take the BC curve $\Gamma \supset \gamma$. Let $z \in \Gamma \backslash \gamma$. Then the inner supporting disk $B_{i}$ at $z$ is disjoint from $\gamma$, so it can be shifted appropriately so that $z$ is contained in its shift, which is still disjoint from $\gamma$.

The simple example $E=\{i / n\} \cup\{1 / n\} \cup\{0\}, n \geq 1$, exhibits a set $E$, which is not $r$-convex, but such that $\mathbb{C} \backslash E$ satisfies the uniform ball condition.

Given a compact set $E$, consider the unbounded open set $\Omega_{t}:=\{z \in \mathbb{C}$ : $d(z, E)>t\}$ for $t \geq 0$. It is clear that $\left\{\Omega_{t}\right\}$ forms a monotone decreasing family of sets.

Let $\Theta_{t} \subseteq \Omega_{t}$ be the unbounded component of $\Omega_{t}$. It is not hard to construct a compact set $E$ so that $\Theta_{0}=\Omega_{0}$, i.e., $\mathbb{C} \backslash E$ is connected, but $\Theta_{t} \neq \Omega_{t}$ for all $t>0$. We show that this is not the case for $r$-convex sets, and the situation is stable for small enough $t$.

Theorem 2.9. Let $E$ be an $r$-convex compact set for $r>0$, and let $\Theta_{0}=\Omega_{0}$. Then there is $t_{0}=t_{0}(E)$ such that $0<t_{0} \leq r / 4$, and $\Theta_{t}=\Omega_{t}$ for $0 \leq t \leq t_{0}$.

Proof. Define $S=S(E):=\max _{\zeta \in E}|\zeta|$, and assume that $r \leq S(E)$. The proof is split into several steps.

Step 1 . The set $\hat{\Omega}:=\Omega_{r} \cap B(0,2 S)$ is relatively compact, so it contains a finite $r / 2$-net $Z=\left\{z_{j}\right\}_{j=1}^{N}$,

$$
Z \subset \hat{\Omega}, \quad \operatorname{dist}\left(z^{\prime}, Z\right) \leq \frac{r}{2}<r, \quad \forall z^{\prime} \in \hat{\Omega} .
$$

Since $\Omega_{0}=\Theta_{0}=\mathbb{C} \backslash E$ is connected, we can find paths $\Gamma_{j}:[0, \infty) \rightarrow \Theta_{0}$ with

$$
\Gamma_{j}(0)=z_{j}, \quad \Gamma_{j}(\tau) \rightarrow \infty, \quad \tau \rightarrow \infty ; \quad j=1, \ldots, N .
$$

We put $\delta:=\frac{1}{2} \min _{j, \tau} \operatorname{dist}\left(\Gamma_{j}(\tau), E\right)>0$, so that $\Gamma_{j} \subset \Theta_{\delta}, 1 \leq j \leq N$. 
Step 2. Let $z^{\prime} \in \hat{\Omega}$, then there is $z_{k} \in Z, 1 \leq k \leq N$ such that $\left|z^{\prime}-z_{k}\right|<r$. In other words, $z^{\prime} \in B\left(z_{k}, r\right), z_{k} \in B\left(z^{\prime}, r\right)$. Put

$$
B_{1}:=B\left(z_{k}, r\right) \cup B\left(z^{\prime}, r\right), \quad\left\{\xi_{ \pm}\right\}:=\partial B\left(z_{k}, r\right) \cap \partial B\left(z^{\prime}, r\right) .
$$

Since both $z^{\prime}$ and $z_{k}$ are in $\Omega_{r}$, the closure $\bar{B}_{1}$ is contained in $\Omega_{0}$, so

$$
\begin{aligned}
\operatorname{dist}\left(\left[z^{\prime}, z_{k}\right], E\right) & >\operatorname{dist}\left(\left[z^{\prime}, z_{k}\right], \partial B_{1}\right)=\operatorname{dist}\left(\left[z^{\prime}, z_{k}\right],\left\{\xi_{ \pm}\right\}\right) \\
& =\sqrt{r^{2}-\left|z^{\prime}-z_{k}\right|^{2} / 4}>\frac{\sqrt{3}}{2} r .
\end{aligned}
$$

Now, take $t_{0}:=\min (\delta, r / 4)$, so by Step $1,\left[z^{\prime}, z_{k}\right] \subset \Omega_{t}$ and $\Gamma_{k} \subset \Omega_{t}$ for $t \leq t_{0}$. Hence $\left[z^{\prime}, z_{k}\right] \cup \Gamma_{k} \subset \Omega_{t}$, and as the set on the left-hand side is a path from $z^{\prime}$ to infinity, we conclude

$$
\left[z^{\prime}, z_{k}\right] \cup \Gamma_{k} \subset \Theta_{t} \Rightarrow z^{\prime} \in \Theta_{t}, \quad \forall t \leq t_{0} .
$$

Clearly, $B^{c}(0,2 S) \subset \Theta_{t}$ for such $t$, so, finally, $\Omega_{r} \subset \Theta_{t}$ for $t \leq t_{0}$.

Step 3. Assume that for some $\eta, 0<\eta \leq t_{0}$, the statement of the theorem is wrong, so $\Omega_{\eta}$ has a bounded component $D$ such that $D \cap \Theta_{\eta}=\emptyset$. We want to show that

$$
d(z) \leq \sqrt{2} \eta, \quad \forall z \in D .
$$

Let $z \in D$. Note that $d(z) \leq r$, for otherwise $z \in \Omega_{r} \subset \Theta_{\eta}$ by Step 2, and hence $z \in D \cap \Theta_{\eta}$, which is impossible. By the definition of $r$-convexity there exists $z^{\prime} \in \Omega$ such that $z \in B\left(z^{\prime}, r\right) \subset \Omega_{0}$, so $z^{\prime} \in \Omega_{r} \subset \Theta_{\eta}$, and, in particular, $z^{\prime} \neq z$. Hence the segment $\left[z^{\prime}, z\right]$ meets the boundary $\partial D$, so there is a point $\zeta \in\left[z^{\prime}, z\right]$ with $d(\zeta)=\eta$, and we conclude that

$$
\operatorname{dist}\left(\left[z^{\prime}, z\right], E\right) \leq \eta \text {. }
$$

We examine the configuration of two disks, $B\left(z^{\prime}, r\right)$ and $B(z, d(z))$, each of which belongs to $\Omega_{0}$. As the circle $\partial B(z, d(z))$ contains points from $E$, it is clear that the closed disk $\overline{B(z, d(z))}$ cannot lie inside $B\left(z^{\prime}, r\right)$. Hence either the smaller disk $B(z, d(z))$ touches the bigger one from within, and in this case the point $\zeta$ of contact is in $E$ (which implies $d(z)=\operatorname{dist}\left(\left[z^{\prime}, z\right], E\right)$, and (2.5) follows from (2.6)), or the disks have a proper intersection. Write

$$
B_{2}:=B\left(z^{\prime}, r\right) \cup B(z, d(z)) \subset \Omega_{0}, \quad\left\{\xi_{ \pm}\right\}:=\partial B\left(z^{\prime}, r\right) \cap \partial B(z, d(z)) .
$$

Then

$$
\operatorname{dist}\left(\left[z^{\prime}, z\right], E\right) \geq \operatorname{dist}\left(\left[z^{\prime}, z\right], \partial B_{2}\right)=\operatorname{dist}\left(\left[z^{\prime}, z\right], \xi_{+}\right)=\operatorname{dist}\left(\left[z^{\prime}, z\right], \xi_{-}\right) \geq h,
$$

where $h$ is the length of the altitude from the vertex $\xi_{+}$in the triangle $\Delta\left(z^{\prime}, z, \xi_{+}\right)$. If this altitude crosses the side $\left[z^{\prime}, z\right]$ then

$$
\begin{aligned}
& \sqrt{r^{2}-h^{2}}+\sqrt{d^{2}(z)-h^{2}}=\left|z-z^{\prime}\right|<r, \\
& 2 h^{2}>d^{2}(z)+2 \sqrt{\left(r^{2}-h^{2}\right)\left(d^{2}(z)-h^{2}\right)}>d^{2}(z),
\end{aligned}
$$

so $d(z)<\sqrt{2} h$, and (2.5) follows from (2.6) and (2.7). If the altitude crosses the extension of the side $\left[z^{\prime}, z\right]$, one has $d(z)=\operatorname{dist}\left(\left[z^{\prime}, z\right], E\right)$, and again $(2.5)$ holds. 
The inclusion $z \in D \subset \Omega_{\eta}$ means $d(z)>\eta$, so we obtain the two-sided bound

$$
\eta<r^{\prime}:=\sup _{z \in D} d(z) \leq \sqrt{2} \eta \text {. }
$$

Let $\left\{z_{n}\right\} \subset D$ so that $d\left(z_{n}\right) \rightarrow r^{\prime}$. We can assume $z_{n} \rightarrow z_{0}$, and hence there is a point $z_{0} \in D$ with $\eta<d\left(z_{0}\right)=r^{\prime} \leq \sqrt{2} \eta$.

Step 4 . We show that there is a triangle $\Delta(a b c)$ with circumradius $R(\Delta)<4 \eta$ such that

$$
\Delta(a b c) \cap E=\emptyset, \quad E_{1}:=\overline{\Delta(a b c)} \cap E \supset\{a, b, c\} .
$$

Take the point $z_{0}$ from Step 3 and consider the disk $B\left(z_{0}, r^{\prime}\right)$. Its boundary has nonempty intersection with $E$. If the circle $\partial B\left(z_{0}, r^{\prime}\right)$ contains 3 different points from $E$, then in view of $(2.8)$ we are done. Assume that $\partial B\left(z_{0}, r^{\prime}\right) \cap E=\left\{\zeta_{1}\right\}$, or $\partial B\left(z_{0}, r^{\prime}\right) \cap E=\left\{\zeta_{1}, \zeta_{2}\right\}$, and the points $\zeta_{1}$ and $\zeta_{2}$ do not belong to a diameter of the circle. The same argument as in the proof of Proposition 2.4 shows that such configurations cannot occur.

There remains only the case where $z_{0}=0, \partial B\left(z_{0}, r^{\prime}\right) \cap E=\{a, b\}, a=i r^{\prime}$ and $b=-i r^{\prime}$ (after an appropriate affine transformation of the plane). Set

$$
G:=\left\{z=x+i y: 0<x \leq r^{\prime},|y| \leq r^{\prime}\right\} .
$$

Then $G \cap E \neq \emptyset$, since otherwise the circle could be shifted to the right to have $\overline{B\left(z_{0}^{\prime}, r^{\prime}\right)} \cap E=\emptyset$, which, as we have already seen, contradicts the maximality of $r^{\prime}$. Let $h$ be the nonzero number of least magnitude such that the triangle $\Delta\left(a b c_{h}\right)$, $c_{h}=r^{\prime}+i h$, contains points from $E$. The number $h$ exists since by assumption $c_{0}=r^{\prime} \notin E$, and $0<|h| \leq r^{\prime}$ (if there are two options, $h$ and $-h$, we take the positive one). Clearly, such points from $E$ belong to the side $a c_{h}$ for $h>0$ ( $b c_{h}$ for $h<0)$. If we choose the point $c \in E$ on the corresponding side, then $(2.9)$ holds. The triangle $\Delta(a b c)$ is either acute or rectangular. For its sides we have by $(2.8)$

$$
M:=\max (|a b|,|a c|,|b c|) \leq \sqrt{5} r^{\prime} \leq \sqrt{10} \eta,
$$

and by the known upper bound for the circumradius of such a triangle $R(\Delta) \leq$ $M<4 \eta$.

Step 5. The choice of $t_{0}=\min (\delta, r / 4)$ implies $R(\Delta)<4 \eta \leq 4 t_{0} \leq r$. By Proposition 2.2 (see Remark 2.3 after its proof), the set $E_{1}$ in (2.9) is not $r$-convex, and $\operatorname{conv}_{r}\left(E_{1}\right) \cap \Delta(a b c) \neq \emptyset$. Hence, $\operatorname{conv}_{r}(E) \cap \Delta(a b c) \neq \emptyset$, which contradicts the $r$ convexity of $E$, and so the assumption made in Step 3 must be wrong for $r \leq S(E)$.

To remove the assumption $r \leq S(E)$, note that if $r>S(E)$, then $E$ is $r_{1}$-convex with $r_{1}=S(E)$. So for the value $t_{0}$ one has $0<t_{0} \leq r_{1} / 4<r / 4$, as needed. The proof is complete.

Note that for $E=\left\{\zeta_{1}, \ldots, \zeta_{N}\right\}$ the result is obvious with

$$
0 \leq t \leq t_{1}(E):=\frac{1}{2} \delta(E), \quad \delta(E):=\min _{i \neq k}\left|\zeta_{i}-\zeta_{k}\right| .
$$




\section{Lower bounds for Green's functions}

We will be dealing with domains $\Omega=\overline{\mathbb{C}} \backslash E$ where $E$ a compact set in $\mathbb{C}$.

Definition 3.1. The Green's function for a domain $\Omega$ is a map $G_{\Omega}: \Omega \times \Omega \rightarrow$ $(-\infty, \infty]$, such that, for each $w \in \Omega$,

(i) $G_{\Omega}(\cdot, w)$ is harmonic on $\Omega \backslash\{w\}$, and bounded from above and below outside each neighborhood of $w$;

(ii) $G_{\Omega}(w, w)=\infty$, and as $z \rightarrow w$,

$$
\begin{aligned}
& G_{\Omega}(z, w)=-\log |z-w|+O(1), \quad w \neq \infty \\
& G_{\Omega}(z, w)=\log |z|+O(1), \quad w=\infty
\end{aligned}
$$

(iii) $G_{\Omega}(z, w) \rightarrow 0$, as $z \rightarrow \zeta$, for nearly every $\zeta \in \partial \Omega$.

Let us list some basic properties of the Green's functions in the form we need them later on (see, e.g., Section 4.4 in [33]):

(1) If $\partial \Omega$ is nonpolar, then there exists a unique Green's function $G_{\Omega}$ for $\Omega$;

(2) $G_{\Omega}(z, w)=G_{\Omega}(w, z)>0$, moreover, if $\Omega^{\prime}$ is a relatively compact (in $\overline{\mathbb{C}}$ ) open subset of $\Omega$, then $\min _{z, w \in \Omega^{\prime}} G_{\Omega}(z, w)=C\left(\Omega, \Omega^{\prime}\right)>0$;

(3) If $\Omega^{\prime} \subset \Omega^{\prime \prime}$ are domains in $\overline{\mathbb{C}}$ with nonpolar boundaries, then

$$
G_{\Omega^{\prime}}(z, w) \leq G_{\Omega^{\prime \prime}}(z, w), \quad z, w \in \Omega^{\prime} .
$$

The notion of Harnack distance proves useful for our reasoning (see [33], pp. 14-15).

Definition 3.2. Let $D$ be a domain in $\overline{\mathbb{C}}$. Given $z_{1}, z_{2} \in D$, the Harnack distance between $z_{1}$ and $z_{2}$ is the smallest number $\tau_{D}\left(z_{1}, z_{2}\right)$ so that for every positive harmonic function $h$ on $D$,

$$
\tau_{D}^{-1}\left(z_{1}, z_{2}\right) h\left(z_{2}\right) \leq h\left(z_{1}\right) \leq \tau_{D}\left(z_{1}, z_{2}\right) h\left(z_{2}\right) .
$$

It is known that

1. $\tau_{D}(z, w)=\tau_{D}(w, z) \geq 1$ and $\tau_{D}(z, z)=1$;

2. $\tau_{D}\left(z_{1}, z_{3}\right) \leq \tau_{D}\left(z_{1}, z_{2}\right) \tau_{D}\left(z_{2}, z_{3}\right)$ for $z_{1}, z_{2}, z_{3} \in D$;

3. $\tau_{D}$ is a continuous function of both variables, in particular, if $D_{1}$ is a relatively compact (in $\overline{\mathbb{C}}$ ) open subset of $D$, then $\max _{z, w \in D_{1}} \tau_{D}(z, w)=C\left(D, D_{1}\right)<\infty$.

Given a compact set $E$, we recall the notation $\Omega_{t}=\{z \in \overline{\mathbb{C}}: d(z)>t\}$ (we view $\Omega_{t}$ as an open subset of $\overline{\mathbb{C}}$ ). If $E$ is an $r$-convex compact set with connected complement, then, by Theorem 2.9, we know that $\Omega_{t}$ is a subdomain of $\overline{\mathbb{C}}$ for sufficiently small $t$. Its boundary $\partial \Omega_{t}=\{z: d(z)=t\}$ is nonpolar (since it splits the plane), so the Green's function $G_{t}$ for $\Omega_{t}$ exists and is unique.

The main technical tool is the following lower bound for $G_{t}(z, \infty)$. In what follows $C=C(E)$ stands for different positive constants which depend only on $E$, and the particular values of which are immaterial. 
Lemma 3.3. Let $E$ be an $r$-convex compact set with connected complement $\Omega=$ $\overline{\mathbb{C}} \backslash E$. Then for $0<t \leq t_{0}$, where $t_{0} \leq r / 4$ is defined in Theorem 2.9,

$$
G_{t / 3}(z, \infty) \geq C \min \{1, d(z)\}, \quad z \in \Omega_{t} .
$$

Proof. By Theorem 2.9, we have $\Omega_{t}=\Theta_{t}$ for $0<t \leq t_{0}$.

Assume first that $d(z)>t_{0}$, so $z \in \Omega_{t_{0}}$. By properties (2) and (3),

$$
G_{t / 3}(z, \infty) \geq G_{t_{0} / 3}(z, \infty) \geq C \geq C \min \{1, d(z)\},
$$

as needed.

For the rest of the proof we assume that $z \in \Omega_{t}$ and $d(z) \leq t_{0}$, so $t<d(z) \leq t_{0}$. By $r$-convexity, $z \in B\left(z^{\prime}, r\right) \subset \Omega$, and since $2 t_{0}<r$ (see Theorem 2.9), the following chain of inequalities can be checked easily:

$$
r>\left|z-z^{\prime}\right| \geq d\left(z^{\prime}\right)-d(z) \geq r-t_{0}>t_{0} \geq d(z)>t .
$$

Define

$$
r_{1}:=\left|z-z^{\prime}\right|-\frac{t}{3}, \quad r_{2}:=d(z)-\frac{t}{3},
$$

so $2 t / 3<r_{2}<r_{1}<\left|z-z^{\prime}\right|$. It follows from (3.2) that the disks $B\left(z^{\prime}, r_{1}\right)$ and $B\left(z, r_{2}\right)$ satisfy

(a) $B\left(z^{\prime}, r_{1}\right) \cup B\left(z, r_{2}\right) \subset \Omega_{t / 3}$;

(b) $z^{\prime} \notin B\left(z, r_{2}\right), \quad z \notin B\left(z^{\prime}, r_{1}\right)$;

(c) $B\left(z^{\prime}, r_{1}\right) \cap B\left(z, r_{2} / 2\right) \neq \emptyset$. Indeed,

$$
r_{1}+\frac{r_{2}}{2}=\left|z-z^{\prime}\right|-\frac{t}{3}+\frac{1}{2}\left(d(z)-\frac{t}{3}\right)>\left|z-z^{\prime}\right| .
$$

Let $L:=\partial B\left(z^{\prime}, r_{1}\right) \cap B\left(z, 3 r_{2} / 4\right)$ be the arc of $\partial B\left(z^{\prime}, r_{1}\right)$ inside $B\left(z, 3 r_{2} / 4\right)$. A simple argument from planar geometry shows that property (c) implies the lower bound $|L|>r_{2} / 2$ for the length of $L$.

We proceed with the bounds for the Green's functions. By properties (a) and (b), the function $G_{t / 3}(\cdot, z)$ is harmonic and positive in the disk $B\left(z^{\prime}, r_{1}\right)$. As $r_{1}<\left|z-z^{\prime}\right|<r$, the mean value theorem provides

$$
G_{t / 3}\left(z^{\prime}, z\right)=\frac{1}{2 \pi r_{1}} \int_{\partial B\left(z^{\prime}, r_{1}\right)} G_{t / 3}(\zeta, z) m(d \zeta) \geq \frac{1}{2 \pi r} \int_{L} G_{t / 3}(\zeta, z) m(d \zeta) .
$$

Since $B\left(z, r_{2}\right) \subset \Omega_{t / 3}$, and the Green's function increases with the domain, we have

$$
G_{t / 3}(u, v) \geq G_{B\left(z, r_{2}\right)}(u, v), \quad u, v \in B\left(z, r_{2}\right) .
$$

The latter can be computed explicitly as

$$
G_{B\left(z, r_{2}\right)}(z, v)=\log \left|\frac{r_{2}}{v-z}\right| \geq \log \frac{4}{3}, \quad v \in B\left(z, \frac{3 r_{2}}{4}\right) .
$$


Hence $G_{t / 3}(z, \zeta) \geq \log \frac{4}{3}$ for $\zeta \in L$, so, taking into account $r_{2}=d(z)-t / 3>$ $2 d(z) / 3$, we obtain the lower bound

$$
G_{t / 3}\left(z^{\prime}, z\right) \geq \frac{\log \frac{4}{3}}{2 \pi r}|L|>\frac{\log \frac{4}{3}}{4 \pi r} r_{2}>\frac{\log \frac{4}{3}}{6 \pi r} d(z) .
$$

To pass from $z^{\prime}$ to $\infty$, we invoke the Harnack distance. Let $D=\Omega_{2 t_{0}}$ be a domain in $\overline{\mathbb{C}}$ which depends only on $E$ and such that $D \subset \Omega_{t / 3}$. It is clear that $z \notin D$ (by the assumption $d(z) \leq t_{0}$ ), so the function $h_{t, z}(\zeta):=G_{t / 3}(z, \zeta)$ is positive and harmonic in $D$. Next, $2 t_{0}<r$ implies $\Omega_{r}$ is a relatively compact subset of $D$, so $z^{\prime} \in \Omega_{r} \subset D$, and, by the definition of the Harnack distance with $z_{1}=\infty$ and $z_{2}=z^{\prime}$,

$$
\tau_{D}^{-1}\left(\infty, z^{\prime}\right) G_{t / 3}\left(z, z^{\prime}\right) \leq G_{t / 3}(z, \infty)
$$

By property (3) of the Harnack distance, $\min _{z^{\prime} \in \Omega_{r}} \tau_{D}^{-1}\left(\infty, z^{\prime}\right)=C>0$, and hence, by $(3.3)$,

$$
G_{t / 3}(z, \infty) \geq C d(z) \geq C \min \{1, d(z)\}, \quad z \in \Omega_{t},
$$

as claimed. The proof is complete.

Remark 3.4. Assume that $E$ is a nonpolar $r$-convex compact set with connected complement. Then the Green's function $G=G_{0}$ exists and is unique, and it easily follows from Lemma 3.3 that

$$
G(z, \infty) \geq C \min \{1, d(z)\}, \quad z \in \Omega .
$$

For similar bounds for the Green's functions of a bounded domain with $C^{2}$ boundary, see formula (2.8) in [38]. Note that our result is proved under no assumptions on the smoothness of the boundary.

\section{Proof of the main result and its consequences}

We consider subharmonic functions and their Riesz measures. Let $\mathcal{D}$ be a domain in $\overline{\mathbb{C}}$ such that its boundary $\partial \mathcal{D}$ is nonpolar, and let $v$ be a subharmonic function on $\mathcal{D}, v \neq \equiv-\infty$, which has a harmonic majorant on $\mathcal{D}$. Let $\mu=1 /(2 \pi) \Delta v$ be its Riesz measure. By the fundamental Riesz decomposition theorem (RDT) (see, e.g., Theorem 4.5.4 in [33])

$$
v(z)=u(z)-\int_{\mathcal{D}} G(z, \zeta) \mu(d \zeta), \quad z \in \mathcal{D},
$$

$u$ is the least harmonic majorant of $v$ on $\mathcal{D}$ and $G$ is the Green's function of $\mathcal{D}$.

We apply this result to subharmonic functions on $\Omega=\overline{\mathbb{C}} \backslash E$ where $E$ is an $r$-convex compact set, with $\mathcal{D}=\Omega_{t}$ for $t \leq t_{0}$ from Lemma 3.3, so its boundary is nonpolar, and $G=G_{t}$. We assume that the subharmonic function $v$ on $\Omega$ is subject to the growth and normalization conditions (1.3), and we assume that $\psi$ 
is a positive and monotone decreasing function on $\mathbb{R}_{+}$. Hence $v$ has a harmonic majorant $u_{t}$ on $\mathcal{D}$, and so

$$
v(z)=u_{t}(z)-\int_{\Omega_{t}} G_{t}(z, \zeta) \mu(d \zeta), \quad z \in \Omega_{t} .
$$

Proof of Theorem 1.2. By (1.3), $v$ is bounded above on $\Omega_{t}$, and

$$
v(z) \leq K_{v} \psi(t), \quad z \in \Omega_{t}, \quad t>0,
$$

so the least harmonic majorant $u_{t}$ exists and satisfies the same bound (4.2). Moreover, (4.1) with $z=\infty$ gives

$$
\int_{\Omega_{t}} G_{t}(\infty, \zeta) \mu(d \zeta) \leq K_{v} \psi(t), \quad 0<t \leq t_{0} .
$$

Next, it follows from the hypothesis of the theorem that $\varphi_{1}^{\prime} \geq 0$ a.e. on some interval $[0, \delta]$ (we assume $\delta \leq t_{0}$ ). We decompose the integral on the left-hand side of (1.5) as

$$
\begin{aligned}
\int_{\Omega} \varphi(d(\zeta)) \mu(d \zeta) & \leq\left\{\int_{\Omega \backslash \Omega_{\delta}}+\int_{\Omega_{\delta} \cap B(0,6 S+1)}+\int_{B^{c}(0,6 S+1)}\right\} \varphi(d(\zeta)) \mu(d \zeta) \\
& =I_{1}+I_{2}+I_{3}, \quad S=\max _{\zeta \in E}|\zeta| .
\end{aligned}
$$

We begin with the bound for

$$
I_{1}=\int_{\Omega \backslash \Omega_{\delta}} \varphi_{1}(d(\zeta)) \sigma(d \zeta), \quad \sigma(d \zeta):=d(\zeta) \mu(d \zeta) .
$$

Put

$$
H_{\delta}(t):=\int_{\Omega_{t} \backslash \Omega_{\delta}} d(\zeta) \mu(d \zeta)=\int_{\Omega_{t} \backslash \Omega_{\delta}} \sigma(d \zeta)=\sigma(\{\zeta: t<d(\zeta) \leq \delta\})
$$

The following result known as the layer cake representation (LCR) theorem (see Theorem 1.13 in [26]), plays a key role in the next step of the proof.

Theorem (LCR). Let $\nu$ be a measure on the Borel sets of the positive real line $\mathbb{R}_{+}$ such that

$$
\xi(t):=\nu([0, t)), \quad \xi(0)=0
$$

is finite for every $t>0$. Let $(X, \Sigma, \sigma)$ be a measure space and let $f$ be any nonnegative measurable function on $X$. Then

$$
\int_{X} \xi(f(x)) \sigma(d x)=\int_{0}^{\infty} \sigma(\{x: f(x)>t\}) \nu(d t) .
$$

We apply this with $X=\Omega \backslash \Omega_{\delta}, \nu(d t)=\varphi_{1}^{\prime}(t) d t$ and $f(x)=d(x)$ :

$$
I_{1}=\int_{\Omega \backslash \Omega_{\delta}} \varphi_{1}(d(\zeta)) \sigma(d \zeta)=\int_{0}^{\delta} \varphi_{1}^{\prime}(t) H_{\delta}(t) d t .
$$


For $t<d(\zeta) \leq \delta$ one has $d(\zeta) \leq(1+\delta) \min \{1, d(\zeta)\} \leq C G_{t / 3}(\zeta, \infty)$ in view of Lemma 3.3. Here and in the rest of the proof $C$ stands for a positive constant which depends on $E, \varphi$, and $\psi$, as in (1.5). So by (4.3),

$$
H_{\delta}(t) \leq C \int_{\Omega_{t}} G_{t / 3}(\zeta, \infty) \mu(d \zeta) \leq C \int_{\Omega_{t / 3}} G_{t / 3}(\zeta, \infty) \mu(d \zeta) \leq C K_{v} \psi\left(\frac{t}{3}\right)
$$

and, finally,

$$
I_{1} \leq C K_{v} \int_{0}^{\delta} \varphi_{1}^{\prime}(t) \psi\left(\frac{t}{3}\right) d t
$$

Since $d(\zeta) \leq|\zeta|+S \leq 7 S+1$ for $\zeta \in B(0,6 S+1)$, the bound for $I_{2}$ is

$$
\begin{aligned}
I_{2} & =\int_{\Omega_{\delta} \cap B(0,6 S+1)} \varphi_{1}(d(\zeta)) d(\zeta) \mu(d \zeta) \leq \max _{\delta \leq y \leq 7 S+1} \varphi_{1}(y) \int_{\Omega_{\delta}} d(\zeta) \mu(d \zeta) \\
& \leq C \int_{\Omega_{\delta}} \min \{1, d(\zeta)\} \mu(d \zeta) \leq C \int_{\Omega_{\delta / 3}} G_{\delta / 3}(\zeta, \infty) \mu(d \zeta) \leq C K_{v} \psi\left(\frac{\delta}{3}\right),
\end{aligned}
$$

and so

$$
I_{2} \leq C K_{v}
$$

The bound for $I_{3}$ is standard, and has nothing to do with the subtle Lemma 3.3. The LCR theorem and the inclusion $B^{c}(0,6 S+1) \subset \Omega_{5 S+1}$ give

$$
I_{3} \leq \int_{\Omega_{5 S+1}} \varphi(d(\zeta)) \mu(d \zeta)=\int_{5 S+1}^{\infty} \varphi^{\prime}(t) H(t) d t, \quad H(t):=\int_{\Omega_{t}} \mu(d \zeta) .
$$

For $t \geq 5 S+1$ we put

$$
R_{t}:=\frac{2}{3}(t-S) \geq \frac{2}{3}(4 S+1), \quad R_{t}-S=\frac{2 t-5 S}{3} \geq \frac{t}{3},
$$

and apply again the RDT in the form

$$
v(z)=\tilde{u}(z)-\int_{|\zeta|>R_{t}} \tilde{G}(z, \zeta) \mu(d \zeta), \quad|z|>R_{t},
$$

where $\tilde{G}$ is the Green's function of the domain $\left\{\zeta:|\zeta|>R_{t}\right\}$ and $\tilde{u}$ is the least harmonic majorant of $v$ on this domain. Since $d(z) \geq R_{t}-S$ for $|z|>R_{t}$, the assumptions on $v$ and $\psi$ imply

$$
\tilde{u}(z) \leq K_{v} \psi\left(R_{t}-S\right), \quad|z|>R_{t}
$$

and, as above,

$$
\int_{|\zeta|>R_{t}} \tilde{G}(\infty, \zeta) \mu(d \zeta) \leq K_{v} \psi\left(R_{t}-S\right) \leq K_{v} \psi\left(\frac{t}{3}\right)
$$


The function $\tilde{G}(\infty, \zeta)$ is known to have the explicit form $\tilde{G}(\infty, \zeta)=\log |\zeta|-\log \left|R_{t}\right|$, so, by (4.7), we conclude

$$
\begin{aligned}
\log \frac{3}{2} \int_{|\zeta|>\frac{3}{2} R_{t}} \mu(d \zeta) & \leq \int_{|\zeta|>\frac{3}{2} R_{t}} \log \left|\frac{\zeta}{R_{t}}\right| \mu(d \zeta) \\
& \leq \int_{|\zeta|>R_{t}} \log \left|\frac{\zeta}{R_{t}}\right| \mu(d \zeta) \leq K_{v} \psi\left(\frac{t}{3}\right) .
\end{aligned}
$$

Next, note that

$$
\left\{\zeta:|\zeta|>\frac{3}{2} R_{t}\right\} \supset\left\{\zeta: d(\zeta)>\frac{3}{2} R_{t}+S\right\}=\Omega_{t}
$$

so

$$
H(t)=\int_{\Omega_{t}} \mu(d \zeta) \leq C K_{v} \psi\left(\frac{t}{3}\right)
$$

and, finally, in view of (4.6), we have

$$
I_{3} \leq C K_{v} \int_{5 S+1}^{\infty} \varphi^{\prime}(t) \psi\left(\frac{t}{3}\right) d t
$$

The main statement of Theorem 1.2 now follows from (4.4), (4.5), and (4.8).

The case of bounded subharmonic functions is not formally covered by the main result since $\varphi_{1}(0)=1 \neq 0$ for $\varphi(x)=\min \{1, x\}$. Fortunately, (1.6) is a direct consequence of (4.3) and Lemma 3.3. Indeed, for $0<t<t_{0}$

$$
C K_{v} \geq \int_{\Omega_{t}} G_{t}(\infty, \zeta) \mu(d \zeta) \geq C \int_{\Omega_{t}} \min \{1, d(\zeta)\} \mu(d \zeta),
$$

and there remains only to let $t \rightarrow 0$. The proof is complete.

Corollary 4.1. Suppose that for a subharmonic function $v$ estimate (1.3) holds with $\psi(t)=t^{-q}, q>0$. Then, for each $\varepsilon>0$,

$$
\int_{\Omega} \varphi(d(\zeta)) \mu(d \zeta) \leq C(E, q, \varepsilon) K_{v}
$$

with

$$
\varphi(x)=x^{q+1 / 2}(\min \{x, 1 / x\})^{\varepsilon+1 / 2}=\left\{\begin{array}{ll}
x^{q+1+\varepsilon}, & x \leq 1, \\
x^{q-\varepsilon}, & x>1,
\end{array} \quad 0<\varepsilon<q .\right.
$$

In some instances, in addition to the hypothesis of Theorem 1.2, the support of the Riesz measure $\mu$ is bounded. Such a situation occurs when $v=\log |f|$ for an analytic function $f$ on $\Omega$ satisfying $f(\infty)=1$ (see Section 5 ). Now only the first term in (1.4) matters, so we obtain: 
Corollary 4.2. In addition to the assumptions of Theorem 1.2 , let $\operatorname{supp} \mu \subset$ $B\left(0, R_{\mu}\right)$, and, instead of $(1.4)$, suppose

$$
\int_{0} \varphi_{1}^{\prime}(t) \psi\left(\frac{t}{3}\right) d t<\infty
$$

Then

$$
\int_{\Omega} \varphi(d(\zeta)) \mu(d \zeta) \leq C\left(E, \psi, \varphi, R_{\mu}\right) K_{v}
$$

Consider the case of finite sets $E$, where the bound for the Green's function in (3.1) and the main result can be refined. We formulate this for the special bound as in Corollary 4.1, although the general case of Theorem 1.2 can be handled in the same fashion.

Theorem 4.3. Let $E=\left\{\zeta_{1}, \ldots, \zeta_{N}\right\}$ be a finite set and let $v$ be a subharmonic function on $\Omega=\overline{\mathbb{C}} \backslash E$ so that

$$
v(z) \leq \frac{K_{v}}{d^{q}(z)}, \quad v(\infty)=0 ; \quad q>0, \quad z \in \Omega .
$$

Then there are positive constants $k=k(E)>1$ and $t_{2}=t_{2}(E)$, defined below in (4.16), such that

$$
G_{t}(z, \infty)>\frac{\log 2}{N}>0, \quad z \in \Omega_{k t}, \quad t \leq t_{2},
$$

and for each $0<\varepsilon<q$

$$
\int_{\Omega} \varphi(d(\zeta)) \mu(d \zeta) \leq C(E, q, \varepsilon) K_{v}, \quad \varphi(x)= \begin{cases}x^{q+\varepsilon}, & x \leq 1 \\ x^{q-\varepsilon}, & x>1\end{cases}
$$

If, in addition, $\operatorname{supp} \mu$ is bounded then

$$
\int_{\Omega} d^{q+\varepsilon}(\zeta) \mu(d \zeta)<\infty
$$

Proof. Put

$$
m_{j}:=\prod_{i \neq j}\left|\zeta_{i}-\zeta_{j}\right|, \quad C:=2^{N-1} \max _{j} m_{j}
$$

The function

$$
v_{t}(z):=\frac{1}{N}\left(\sum_{j=1}^{N} \log \left|z-\zeta_{j}\right|-\log t-\log C\right)
$$

is subharmonic on $\mathbb{C}$ (and harmonic on $\Omega$ ), and $v_{t}(z)=\log |z|+O(1)$, as $z \rightarrow \infty$. For

$$
0 \leq t \leq t_{1}(E)=\frac{1}{2} \delta(E), \quad \delta(E):=\min _{i \neq k}\left|\zeta_{i}-\zeta_{k}\right|
$$


(see (2.11)), on each circle $\left|z-\zeta_{n}\right|=t, 1 \leq n \leq N$, one has

$$
v_{t}(z)=\frac{1}{N}\left(\sum_{j \neq n} \log \left|z-\zeta_{j}\right|-\log C\right) .
$$

Since $\left|z-\zeta_{j}\right| \leq\left|z-\zeta_{n}\right|+\left|\zeta_{n}-\zeta_{j}\right|=t+\left|\zeta_{n}-\zeta_{j}\right|$,

$$
\begin{aligned}
v_{t}(z) & \leq \frac{1}{N}\left(\sum_{j \neq n} \log \left(\left|\zeta_{j}-\zeta_{n}\right|+t\right)-\log C\right) \\
& \leq \frac{1}{N}\left((N-1) \log 2+\sum_{j \neq n} \log \left|\zeta_{n}-\zeta_{j}\right|-\log C\right) \leq 0
\end{aligned}
$$

in view of the choice of $C$. Hence $u_{t}(z)=v_{t}(z)-G_{t}(z, \infty)$ is subharmonic on $\Omega_{t}$ and

$$
\limsup _{z \rightarrow \zeta} u_{t}(z) \leq 0, \quad \zeta \in \partial \Omega_{t}, \quad \limsup _{z \rightarrow \infty} \frac{u_{t}(z)}{\log |z|}=0,
$$

so by the Phragmen-Lindelöf principle (see Corollary 2.3.3 in [33]) $u_{t} \leq 0$, or

$$
v_{t}(z) \leq G_{t}(z, \infty), \quad z \in \Omega_{t} .
$$

On the other hand, put

$$
k:=1+2 C\left(\frac{2}{\delta(E)}\right)^{N-1}>1, \quad t_{2}:=\frac{t_{1}}{k} .
$$

For $z \in \Omega_{k t}$ and $t \leq t_{2}$ we have for some $l, m, 1 \leq l, m \leq n$, that

$$
\min _{1 \leq i \leq n}\left|z-\zeta_{i}\right|=\left|z-\zeta_{l}\right|>k t, \min _{i \neq l}\left|z-\zeta_{i}\right|=\left|z-\zeta_{m}\right| \geq\left|\zeta_{l}-\zeta_{m}\right|-\left|z-\zeta_{l}\right| \geq \delta(E)-k t,
$$

so

$$
\begin{aligned}
v_{t}(z) & =\frac{1}{N}\left(\sum_{j \neq l} \log \left|z-\zeta_{j}\right|+\log \left|z-\zeta_{l}\right|-\log t-\log C\right) \\
& >\frac{1}{N}((N-1) \log (\delta(E)-k t)+\log k t-\log t-\log C) \\
& \geq \frac{1}{N}\left((N-1) \log \frac{\delta(E)}{2}+\log k-\log C\right)>\frac{\log 2}{N},
\end{aligned}
$$

by the choice of $k$ and $C$. Finally,

$$
G_{t}(z, \infty) \geq v_{t}(z)>\frac{\log 2}{N}>0, \quad z \in \Omega_{k t}, \quad t \leq t_{2},
$$

as needed.

The rest of the proof follows the same line of reasoning as the the proof of Theorem 1.2, using the LCR theorem, with Lemma 3.3 replaced with (4.11). 
To show that Corollary 4.1 and Theorem 4.3 are optimal in some sense, we proceed with the following simple result.

Lemma 4.4. Let $E \subset \mathbb{C}$ be an arbitrary compact set, which does not split the plane, let $D$ be a relatively compact (in the sense of $\overline{\mathbb{C}}$ ) subdomain of $\Omega=\overline{\mathbb{C}} \backslash E$, and suppose $\infty \in D$. Let $v$ be a subharmonic and continuous (in the sense of $\overline{\mathbb{C}}$ ), nonnegative function on $\Omega$. Then the least harmonic majorant $u$ for $D$ exists, and

$$
v_{\min }:=\min _{\zeta \in \partial D} v(\zeta) \leq u(z) \leq \max _{\zeta \in \partial D} v(\zeta)=: v_{\max }, \quad z \in D
$$

Proof. By assumption, $v$ is nonnegative and bounded on $D$, so the least harmonic majorant $u$ exists, and it is nonnegative and bounded.

To prove the right inequality, note that $v$ is continuous on $\bar{D}$, and so

$$
\limsup _{z \rightarrow \zeta \in \partial D} v(z)=v(\zeta) \leq v_{\max }
$$

By the maximum principle $v \leq v_{\max }$, so $u \leq v_{\max }$.

To prove the left inequality, note that

$$
\liminf _{z \rightarrow \zeta} u(z) \geq \liminf _{z \rightarrow \zeta} v(z)=v(\zeta) \geq v_{\min }
$$

Put $V=-u+v_{\min }$. This is a harmonic and bounded function on $D$, and $\limsup _{z \rightarrow \zeta} V(z) \leq 0, \zeta \in \partial D$. Again, by the maximum principle, $V \leq 0$ in $D$, as needed. The proof is complete.

For the class of subharmonic functions $v$ satisfying (1.3) with $\psi(t)=t^{-q}, q>0$, there is an obvious extremal element $\hat{v}(z)=d^{-q}(z)$. This function is subharmonic and continuous on $\Omega$, and it is quite natural to expect that it provides divergence of integrals in (4.9).

We apply Lemma 4.4 to $\hat{v}$. By the RDT,

$$
0=\hat{v}(\infty)=\hat{u}(\infty)-\int_{D} G_{D}(z, \infty) \hat{\mu}(d z), \quad \hat{\mu}=\frac{1}{2 \pi} \Delta \hat{v},
$$

and so, by Lemma 4.4,

$$
\left[\max _{\zeta \in \partial D} d(\zeta)\right]^{-q} \leq \int_{D} G_{D}(z, \infty) \hat{\mu}(d z) \leq\left[\min _{\zeta \in \partial D} d(\zeta)\right]^{-q} .
$$

Two types of domains $D$ are of particular interest.

1. As above in Section 2, let $\Theta_{t}$ be the unbounded component of the set $\Omega_{t}=\{z: d(z)>t\}$. Then $d(\zeta)=t$ on $\partial \Theta_{t}$, so, by (4.18),

$$
\int_{\Theta_{t}} G_{\Theta_{t}}(z, \infty) \hat{\mu}(d z)=t^{-q}
$$

2. Let $D=D_{t}=\{|z|>t\}$ where $t>S=\max _{\zeta \in E}|\zeta|$. Then, for $|z| \geq t$,

$$
\frac{t-S}{t}|z| \leq d(z) \leq|z|+S
$$


$G_{D_{t}}(z, \infty)=\log \frac{|z|}{t}$, and (4.18) takes the form

$$
(t+S)^{-q} \leq \int_{D_{t}} \log \frac{|z|}{t} \hat{\mu}(d z) \leq(t-S)^{-q} .
$$

We mention two important consequences of (4.21). First, let $t>\tau>S$. Then

$$
\begin{aligned}
\int_{D_{t}} \hat{\mu}(d z) & \leq\left(\log \frac{t}{\tau}\right)^{-1} \int_{D_{t}} \log \frac{|z|}{\tau} \hat{\mu}(d z) \leq\left(\log \frac{t}{\tau}\right)^{-1} \int_{D_{\tau}} \log \frac{|z|}{\tau} \hat{\mu}(d z) \\
& \leq\left(\log \frac{t}{\tau}\right)^{-1}(\tau-S)^{-q}<\infty .
\end{aligned}
$$

Next,

$$
\int_{D_{t}} \log |z| \hat{\mu}(d z) \leq(t-S)^{-q}+\log t \int_{D_{t}} \hat{\mu}(d z)<\infty .
$$

We show now that Corollary 4.1 is false for the function $\hat{v}$ and $\varepsilon<0$.

Theorem 4.5. Let $E \subset \mathbb{C}$ be an arbitrary compact set, which does not split the plane and let $\hat{v}(z)=d^{-q}(z), q>0$. Then, for each $0<\varepsilon<q$,

$$
I_{ \pm}:=\int_{\Omega} d^{q \pm \varepsilon}(z) \hat{\mu}(d z)=+\infty .
$$

Proof. Define $M:=B(0, S+1) \backslash E=B(0, S+1) \bigcap \Omega$. We actually prove that

$$
\int_{D_{S+1}} d^{q+\varepsilon}(z) \hat{\mu}(d z)=\int_{M} d^{q-\varepsilon}(z) \hat{\mu}(d z)=+\infty, \quad D_{S+1}=\{|z|>S+1\} .
$$

We begin with $I_{+}$. By (4.20) with $t=S+1$ we have, for $|z| \geq S+1$,

$$
d^{q+\varepsilon}(z) \geq \frac{|z|^{q+\varepsilon}}{(S+1)^{q+\varepsilon}} \geq C_{1}(E, q, \varepsilon)|z|^{q} \log |z|,
$$

so that

$$
\int_{D_{S+1}} d^{q+\varepsilon}(z) \hat{\mu}(d z) \geq C_{1}(E, q, \varepsilon) \int_{D_{S+1}}|z|^{q} \log |z| \hat{\mu}(d z) .
$$

Let $\sigma(d z)$ be the restriction of $\log |z| \hat{\mu}(d z)$ to $D_{S+1}$. The LCR theorem gives

$$
\begin{aligned}
\int_{D_{S+1}}|z|^{q} \sigma(d z) & =q \int_{0}^{\infty} t^{q-1} d t \int_{D_{t} \cap D_{S+1}} \log |z| \hat{\mu}(d z) \\
& =(S+1)^{q} \int_{D_{S+1}} \log |z| \hat{\mu}(d z)+q \int_{S+1}^{\infty} t^{q-1} d t \int_{D_{t}} \log |z| \hat{\mu}(d z),
\end{aligned}
$$

so, by (4.25),

$$
\int_{D_{S+1}} d^{q+\varepsilon}(z) \hat{\mu}(d z) \geq C_{2}(E, q, \varepsilon) \int_{S+1}^{\infty} t^{q-1} d t \int_{D_{t}} \log |z| \hat{\mu}(d z) .
$$


But $G_{D_{t}}(z, \infty)=\log |z|-\log t<\log |z|$, and it follows from (4.21) that

$$
\int_{D_{t}} \log |z| \hat{\mu}(d z) \geq \int_{D_{t}} G_{D_{t}}(z, \infty) \hat{\mu}(d z) \geq(t+S)^{-q},
$$

which implies

$$
I_{+} \geq \int_{D_{S+1}} d^{q+\varepsilon}(z) \hat{\mu}(d z)=+\infty
$$

as claimed.

The domain $\Theta_{x}$ plays a key role in estimating $I_{-}$. Let $z \in \Theta_{x}$. Then for every $z_{0} \in E$ one has

$$
\left|z-z_{0}\right| \geq d(z)>x, \quad \frac{\left|z-z_{0}\right|}{x}>1
$$

so the function $h(z)=\log \left(\left|z-z_{0}\right| / x\right)$ is harmonic on $\Theta_{x}$ and

$$
h(z) \geq 0, \quad z \in \bar{\Theta}_{x} ; \quad h(z)=\log |z|+O(1), \quad z \rightarrow \infty .
$$

Hence by the maximum principle

$$
\log \frac{\left|z-z_{0}\right|}{x}-G_{\Theta_{x}}(z, \infty) \geq 0, \quad z \in \Theta_{x}
$$

Define $M_{x}:=B(0, S+1) \cap \Omega_{x}$ and $N_{x}:=B(0, S+1) \cap \Theta_{x} \subset M_{x}$. If $z \in N_{x}$ and $x<1$, then $(4.26)$ implies

$$
G_{\Theta_{x}}(z, \infty)<\log \frac{2 S+1}{x}<C_{3}(E, \varepsilon) x^{-\varepsilon}
$$

We apply again the LCR theorem to obtain

$$
\begin{aligned}
\int_{M} d^{q-\varepsilon}(z) \hat{\mu}(d z) & =(q-\varepsilon) \int_{0}^{S+1} x^{q-\varepsilon-1} d x \int_{M_{x}} \hat{\mu}(d z) \\
& \geq(q-\varepsilon) \int_{0}^{1} x^{q-\varepsilon-1} d x \int_{N_{x}} \hat{\mu}(d z) .
\end{aligned}
$$

By (4.27),

$$
\int_{M} d^{q-\varepsilon}(z) \hat{\mu}(d z) \geq C_{4}(E, q, \varepsilon) \int_{0}^{1} x^{q-1} d x \int_{N_{x}} G_{\Theta_{x}}(z, \infty) \hat{\mu}(d z) .
$$

Next,

$$
\int_{N_{x}} G_{\Theta_{x}}(z, \infty) \hat{\mu}(d z)=\int_{\Theta_{x}} G_{\Theta_{x}}(z, \infty) \hat{\mu}(d z)-\int_{\Theta_{x} \cap \bar{D}_{S+1}} G_{\Theta_{x}}(z, \infty) \hat{\mu}(d z) .
$$

The first integral on the right-hand side equals $x^{-q}$ due to (4.19). For the second one, we have, by (4.26), (4.22), and (4.23),

$$
\begin{gathered}
\int_{\Theta_{x} \cap \bar{D}_{S+1}} G_{\Theta_{x}}(z, \infty) \hat{\mu}(d z) \leq \int_{\Theta_{x} \cap \bar{D}_{S+1}} \log \frac{\left|z-z_{0}\right|}{x} \hat{\mu}(d z) \leq \int_{\bar{D}_{S+1}} \log \frac{2|z|}{x} \hat{\mu}(d z) \\
=\int_{\bar{D}_{S+1}} \log |z| \hat{\mu}(d z)+\log \frac{2}{x} \int_{\bar{D}_{S+1}} \hat{\mu}(d z) \leq C_{5}(E)\left(1+\log \frac{2}{x}\right) .
\end{gathered}
$$


Finally,

$$
\int_{N_{x}} G_{\Theta_{x}}(z, \infty) \hat{\mu}(d z) \geq x^{-q}-C_{5}(E)\left(1+\log \frac{2}{x}\right) \geq C_{6}(E) x^{-q}
$$

for sufficiently small $x$, and so

$$
I_{-} \geq \int_{M} d^{q-\varepsilon}(z) \hat{\mu}(d z)=+\infty
$$

The proof is complete.

It follows from (4.24) (compare with Corollary 4.1) that

$$
\int_{\Omega} \hat{\varphi}(d(z)) \hat{\mu}(d z)=+\infty, \quad \hat{\varphi}(x)= \begin{cases}x^{q-\varepsilon}, & x \leq 1 \\ x^{q+\varepsilon}, & x>1\end{cases}
$$

It turns out that Corollary 4.1 is false for the function $\hat{v}$ even for $\varepsilon=0$ as long as we consider particular sets $E$.

Example. Let $E_{0}=[0,1], v_{0}(z)=d^{-2}\left(z, E_{0}\right)$, and $\mu_{0}=\frac{1}{2 \pi} \Delta v_{0}$. By Corollary 4.1 ,

$$
\int_{\Omega_{0}} \varphi_{0}(d(\zeta)) \mu_{0}(d \zeta)<\infty, \quad \Omega_{0}=\mathbb{C} \backslash E_{0}, \quad \varphi_{0}(x)=\left\{\begin{array}{ll}
x^{3+\varepsilon}, & x \leq 1, \\
x^{2-\varepsilon}, & x>1,
\end{array} \quad \forall \varepsilon>0 .\right.
$$

To show that the integral diverges for $\varepsilon=0$ we compute the Riesz measure $\mu_{0}$ of $v_{0}$ explicitly. Indeed, now $\Omega_{0}=\mathbb{C}_{1} \cup \mathbb{C}_{2} \cup \mathbb{C}_{3}$, where

$\mathbb{C}_{1}=\{z: 0 \leq x \leq 1, y \neq 0\}, \quad \mathbb{C}_{2}=\{z: x<0\}, \quad \mathbb{C}_{3}=\{z: x>1\}, \quad z=x+i y$.

We apply the well-known equality $\Delta|F|^{2}=4\left|F^{\prime}\right|^{2}$, for an analytic function $F$ to obtain

$$
v_{0}(z)=\left\{\begin{array}{ll}
y^{-2}, & z \in \mathbb{C}_{1}, \\
|z|^{-2}, & z \in \mathbb{C}_{2}, \\
|z-1|^{-2}, & z \in \mathbb{C}_{3},
\end{array} \quad \Delta v_{0}= \begin{cases}6 y^{-4}, & z \in \mathbb{C}_{1}, \\
4|z|^{-4}, & z \in \mathbb{C}_{2}, \\
4|z-1|^{-4}, & z \in \mathbb{C}_{3} .\end{cases}\right.
$$

For $p>0$ we have

$$
\int_{\Omega_{0}} d^{p}(z) \mu_{0}(d z)=\sum_{j=1}^{3} \int_{\mathbb{C}_{j}} d^{p}(z) \mu_{0}(d z)
$$

The first integral

$$
I_{1}:=\int_{\mathbb{C}_{1}} d^{p}(z) \mu_{0}(d z)=\frac{6}{\pi} \int_{0}^{1} d x \int_{0}^{\infty} \frac{d y}{y^{4-p}}=+\infty
$$

for $p=3$. The second integral

$$
I_{2}:=\int_{\mathbb{C}_{2}} d^{p}(z) \mu_{0}(d z)=\frac{4}{\pi} \int_{-\infty}^{0} d x \int_{0}^{\infty} \frac{d y}{\left(x^{2}+y^{2}\right)^{2-p / 2}}=+\infty
$$

for $p=2$. The computation for $I_{3}$ is similar.

We complete the section with the converse result for analytic functions (see [13]). 
Proposition 4.6. Let $E$ be a compact subset of $\mathbb{C}$ and let $Z=\left\{z_{n}\right\}$ be a sequence of points in $\Omega=\mathbb{C} \backslash E$ so that

$$
K:=\sum_{n \geq 1} d^{q}\left(z_{n}\right)<\infty, \quad q \geq 1 .
$$

Then there is an analytic function $f$ on $\Omega$ with zero set $Z(f)=Z$ and $f(\infty)=1$, such that

$$
\log |f(z)| \leq \frac{C_{q} K}{d^{q}(z)}
$$

Proof. We begin with the well known Weierstrass prime factor of order $p \geq 0$,

$$
W(z, p)=(1-z) \exp \left(\sum_{k=1}^{p} \frac{z^{k}}{k}\right), \quad p \geq 1, \quad W(z, 0)=1-z, \quad z \in \mathbb{C},
$$

and its bounds (see, e.g., Lemma 4.3.1 in [25])

$$
\begin{gathered}
|W(z, p)-1| \leq|z|^{p+1}, \quad|z| \leq 1, \\
\log |W(z, p)| \leq A_{p}|z|^{p}, \quad|z| \geq \frac{1}{3}, \quad A_{p}=3 e(2+\log (p+1)) .
\end{gathered}
$$

Denote by $e_{n} \in E$ one of the points closest to $z_{n}$, i.e., such that $d\left(z_{n}\right)=\left|z_{n}-e_{n}\right|$. Put

$$
f(z):=\prod_{n \geq 1} W\left(u_{n}(z), p\right), \quad u_{n}(z)=\frac{z_{n}-e_{n}}{z-e_{n}}, z \in \Omega,
$$

where $p \geq 0$ is chosen so that $q-1 \leq p<q$, and write

$$
f(z)=\Pi_{1}(z) \cdot \Pi_{2}(z), \quad \Pi_{j}(z)=\prod_{n \in \Lambda_{j}} W\left(u_{n}(z), p\right), \quad j=1,2,
$$

where

$$
\Lambda_{1}=\Lambda_{1}(z)=\left\{n:\left|u_{n}(z)\right| \leq 1\right\}, \quad \Lambda_{2}=\Lambda_{2}(z)=\left\{n:\left|u_{n}(z)\right|>1\right\} .
$$

Since $u_{n}(z) \rightarrow 0$ for each $z \in \Omega$, the product $\Pi_{2}$ is finite. By (4.29)

$$
\sum_{n \in \Lambda_{1}}\left|W\left(u_{n}(z), p\right)-1\right| \leq \sum_{n \in \Lambda_{1}}\left|u_{n}(z)\right|^{p+1} \leq \sum_{n \in \Lambda_{1}}\left|u_{n}(z)\right|^{q} \leq \frac{K}{d^{q}(z)},
$$

so the product $\Pi_{1}$ converges absolutely and uniformly in $\Omega$. Moreover,

$$
\log \left|\Pi_{1}(z)\right| \leq \sum_{n \in \Lambda_{1}}\left|W\left(u_{n}(z), p\right)-1\right| \leq \frac{K}{d^{q}(z)} .
$$

For the second product, by (4.30),

$$
\log \left|\Pi_{2}(z)\right| \leq A_{p} \sum_{n \in \Lambda_{2}}\left|u_{n}(z)\right|^{p} \leq A_{p} \sum_{n \in \Lambda_{2}}\left|u_{n}(z)\right|^{q} \leq \frac{A_{p} K}{d^{q}(z)},
$$

which proves (4.28). The equality $Z(f)=Z$ is obvious by construction. 


\section{Applications to perturbation theory of linear operators}

We recall some rudiments of the spectral theory of linear operators on Hilbert space, related to the structure of the spectrum (see, e.g., Section IV.5.6 in [24]). A bounded linear operator $T$ on the infinite-dimensional Hilbert space $\mathcal{H}$ is said to be a Fredholm operator if its kernel and cokernel are both finite-dimensional subspaces. A complex number $\lambda$ lies in the essential spectrum $\sigma_{\text {ess }}(T)$ of the operator $T$ if $T-\lambda$ is not a Fredholm operator. The essential spectrum is known to be a nonempty closed subset of the spectrum $\sigma(T)$, and its complement $\mathcal{F}(T)=$ $\mathbb{C} \backslash \sigma_{\text {ess }}(T)$ is called the Fredholm domain of $T$ (it is not necessarily connected). Clearly, the resolvent set $\rho(T)=\mathbb{C} \backslash \sigma(T) \subset \mathcal{F}(T)$.

The set of all isolated eigenvalues of finite algebraic multiplicity is referred to as the discrete spectrum $\sigma_{d}(T)=\left\{\lambda_{j}\right\}$. Each eigenvalue is counted according to its algebraic multiplicity. Although $\sigma_{\text {ess }}(T) \cap \sigma_{d}(T)=\emptyset$, the entire spectrum is not in general exhausted by their union. Indeed, write

$$
\mathcal{F}(T)=\bigcup_{j \geq 0} \mathcal{F}_{j}(T),
$$

where $\mathcal{F}_{j}(T)$ are the connected components of $\mathcal{F}(T)$ and $\mathcal{F}_{0}$ is the unbounded component (the outer domain). Then either $\mathcal{F}_{j} \subset \sigma(T)$, or $\mathcal{F}_{j} \cap \sigma(T) \subset \sigma_{d}(T)$ (the latter always occurs for $j=0)$. That $\mathcal{F}(T)$ is connected $\left(\mathcal{F}(T)=\mathcal{F}_{0}(T)\right)$ implies (the union is disjoint)

$$
\sigma(T)=\sigma_{\mathrm{ess}}(T) \dot{\bigcup} \sigma_{d}(T) .
$$

The fundamental theorem of Weyl (see Theorem IV.5.35 in [24]) is an outstanding result in perturbation theory. Its version for bounded operators states that the essential spectrum is stable under compact perturbations, that is, for any bounded operator $A_{0}$ and compact operator $B$

$$
\sigma_{\mathrm{ess}}(A)=\sigma_{\mathrm{ess}}\left(A_{0}\right), \quad A=A_{0}+B .
$$

Under certain conditions (see below) relation (5.1) holds for the spectrum $\sigma(A)$ of the perturbed operator as well, and all accumulation points of $\sigma_{d}(A)$ belong to $\sigma_{\text {ess }}\left(A_{0}\right)$. We aim here to find the quantitative rate of convergence of the eigenvalues of $A$ in the form

$$
\sum_{\lambda \in \sigma_{d}(A)} \Phi(d(\lambda)) \leq C\|B\|_{\mathcal{S}_{q}}^{q}, \quad d(\lambda)=\operatorname{dist}\left(\lambda, \sigma\left(A_{0}\right)\right), \quad q \geq 1,
$$

provided $B$ is contained in $\mathcal{S}_{q}$, the Schatten-von Neumann operator ideal.

Our main assumptions on the unperturbed operator $A_{0}$ are:

(i) $\sigma_{\text {ess }}\left(A_{0}\right)$ does not split the plane;

(ii) $\sigma\left(A_{0}\right)$ is an $r$-convex compact set;

(iii) The resolvent $R\left(\lambda, A_{0}\right)=\left(A_{0}-\lambda\right)^{-1}$ is subject to the bound

$$
\left\|R\left(\lambda, A_{0}\right)\right\| \leq \Psi(d(\lambda)), \quad \lambda \notin \sigma\left(A_{0}\right),
$$

where $\Psi$ is a monotone decreasing function on $\mathbb{R}_{+}$with $\Psi(0)=\infty$ and $\Psi(\infty)=0$. 
Note that conditions (i) and (ii) are certainly fulfilled whenever $\sigma\left(A_{0}\right) \subset \mathbb{R}$ or $\sigma\left(A_{0}\right) \subset \mathbb{T}$ and $\sigma\left(A_{0}\right) \neq \mathbb{T}$. Condition (iii) is not really a restriction, as one can put

$$
\Psi(x)=\sup \left\{\left\|R\left(\lambda, A_{0}\right)\right\|: \quad d(\lambda) \geq x\right\} .
$$

However such a choice of $\Psi$ is very much implicit. There are many operators, for which (5.3) holds with explicit function $\Psi$. These include hyponormal operators (see Theorem 3.10.2 in [32]) and spectral operators (in the sense of Dunford) of finite degree [11] (with $\Psi(x)=x^{-s}, s>0$ ). For normal operators $A_{0}$ equality holds in (5.3) with $\Psi(x)=x^{-1}$. Another typical example is (see [30], [15])

$$
\Psi(x)=\frac{C_{1}}{x} \exp \left(\frac{C_{2}}{x^{2}}\right) .
$$

A key analytic tool in perturbation theory is the (regularized) perturbation determinant

$$
g_{q}(\lambda):=\operatorname{det}_{\lceil q\rceil}\left(I+B R\left(\lambda, A_{0}\right)\right), \quad B=A-A_{0} \in \mathcal{S}_{q},
$$

where $\lceil q\rceil=\min \{n \in \mathbb{N}: n \geq q\}$, thanks to its properties (see, e.g., [36], Section XI.9 of [10] or Section IV.3 of [16]):

1. $g_{q}$ is analytic on $\overline{\mathbb{C}} \backslash \sigma\left(A_{0}\right), g_{q}(\infty)=1$;

2. $\lambda$ is a zero of $g_{q}$ of multiplicity $k$ if and only if $\lambda \in \sigma_{d}(A) \backslash \sigma\left(A_{0}\right)$ with algebraic multiplicity $k$;

3. $\log \left|g_{q}(\lambda)\right| \leq C_{q}\|B\|_{\mathcal{S}_{q}}^{q}\left\|R\left(\lambda, A_{0}\right)\right\|^{q}, \lambda \in \overline{\mathbb{C}} \backslash \sigma\left(A_{0}\right)$.

We are in a position to present the main spectral consequences of Theorem 1.2 and Corollary 4.2.

Theorem 5.1. Given a bounded linear operator $A_{0}$ subject to conditions (i)-(iii), and $B \in \mathcal{S}_{q}, q \geq 1$, let $\Phi$ be a positive and absolutely continuous function on $[0, \infty)$ such that $\Phi_{1}(t)=t^{-1} \Phi(t)$ is monotone increasing in some neighborhood of the origin, $\Phi_{1}(0)=0$, and

$$
\int_{0} \Phi_{1}^{\prime}(t) \Psi^{q}\left(\frac{t}{3}\right) d t+\int^{\infty} \Phi^{\prime}(t) \Psi^{q}\left(\frac{t}{3}\right) d t<\infty .
$$

Then

$$
\sum_{\lambda \in \sigma_{d}(A)} \Phi(d(\lambda)) \leq C\left(\sigma\left(A_{0}\right), \Psi, \Phi, q\right)\|B\|_{\mathcal{S}_{q}}^{q} .
$$

Proof. Since $\sigma_{\text {ess }}\left(A_{0}\right)=\sigma_{\text {ess }}(A)$ does not split the plane, we see that

$$
\sigma\left(A_{0}\right)=\sigma_{\mathrm{ess}}\left(A_{0}\right) \dot{\bigcup} \sigma_{d}\left(A_{0}\right), \quad \sigma(A)=\sigma_{\mathrm{ess}}(A) \dot{U} \sigma_{d}(A),
$$

and so both $\sigma\left(A_{0}\right)$ and $\sigma(A)$ do not split the plane. 
We apply Theorem 1.2 with $E=\sigma\left(A_{0}\right)$ to the subharmonic function

$$
v(z)=\log \left|g_{q}(z)\right|, \quad z \in \rho\left(A_{0}\right) \cup\{\infty\} .
$$

In view of property (3) of perturbation determinants, and condition (iii), inequality (1.3) holds with $K_{v}=C_{q}\|B\|_{\mathcal{S}_{q}}^{q}$ and $\psi=\Psi^{q}$. The Riesz measure $\mu$ of $v$ is now a discrete and integer-valued measure supported on $Z\left(g_{q}\right)$, and $\mu\{\lambda\}$ equals the multiplicity of the zero of $g_{q}$ at $\lambda$ (the algebraic multiplicity of the eigenvalue $\lambda(A)$ ). The only problem is that in $(5.7) \sigma_{d}\left(A_{0}\right)$ is in general nonempty, and, what is more to the point, the set $\sigma_{d}\left(A_{0}\right) \cap \sigma_{d}(A)$ can be nonempty as well, and this part of $\sigma_{d}(A)$ is not controlled by the zero set of the perturbation determinant. ${ }^{2}$ Anyway, since $\Phi(0)=0$, Theorem 1.2 yields

$$
\sum_{\lambda \in \sigma_{d}(A)} \Phi(d(\lambda))=\sum_{\lambda \in \sigma_{d}(A) \backslash \sigma_{d}\left(A_{0}\right)} \Phi(d(\lambda)) \leq C\left(\sigma\left(A_{0}\right), \Psi, \Phi, q\right)\|B\|_{\mathcal{S}_{q}}^{q},
$$

as claimed.

If $\sigma_{\text {ess }}\left(A_{0}\right)$ splits the plane, then (see Remark 1.3 after Theorem 1.2) we can argue as above, with the resolvent set $\rho\left(A_{0}\right)$ replaced by the outer domain $\mathcal{F}_{0}\left(A_{0}\right)$, to obtain the inequality

$$
\sum_{\lambda \in \sigma_{d}(A) \cap \mathcal{F}_{0}\left(A_{0}\right)} \Phi(d(\lambda)) \leq C\left(\sigma\left(A_{0}\right), \Psi, \Phi, q\right)\|B\|_{\mathcal{S}_{q}}^{q}
$$

The question arises naturally, whether condition (i) can be relaxed to

(i') $\sigma\left(A_{0}\right)$ does not split the plane.

The answer is in general negative. Indeed, assume that for $A_{0}$ we have $\sigma\left(A_{0}\right)=\overline{\mathbb{D}}$, $\sigma_{\text {ess }}\left(A_{0}\right)=\partial \mathbb{D}$ (e.g., $A_{0}$ is adjoint to the shift operator in $H^{2}$ ). Then $A$ can be constructed in such a way that

$$
\sigma(A)=\partial \mathbb{D} \dot{\cup} \sigma_{d}(A)
$$

and the portion of $\sigma_{d}(A)$ inside $\mathbb{D}$ cannot be controlled.

In the perturbation theory setting the support of the Riesz measure (the zero set of the perturbation determinant) is bounded, supp $\mu \subset B\left(0, R_{\mu}\right)$, so Corollary 4.2 applies. It implies that the second term in (5.5) can be dropped. On the other hand, the constant $C$ on the right-hand side depends now on $R_{\mu}$. It is easy to see that the value $R_{\mu}$ is controlled by the operator norm $\|B\|$. Indeed, it is proved in Lemma 8.4.2 of [15] that, under condition (5.3),

$$
\max _{\zeta \in \sigma(A)} d(\zeta) \leq x\left(\Psi,\|B\|^{-1}\right)
$$

\footnotetext{
${ }^{2}$ As a matter of fact, the Weinstein-Aronszajn formula says that the order of a zero (pole) of $g_{p}$ at the point $\lambda \in \sigma_{d}(A)$ equals $\nu(\lambda(A))-\nu\left(\lambda\left(A_{0}\right)\right) \in \mathbb{Z}$, the difference of the algebraic multiplicities of the eigenvalue $\lambda$.
} 
where $x(\Psi, a), a>0$, is the largest solution of the equation $\Psi(x)=a$. So one can take

$$
R_{\mu}=\sup _{\lambda \in \sigma\left(A_{0}\right)}|\lambda|+x\left(\Psi,\|B\|^{-1}\right)
$$

as needed. Hence

$$
\int_{0} \Phi_{1}^{\prime}(t) \Psi^{q}\left(\frac{t}{3}\right) d t<\infty \Longrightarrow \sum_{\lambda \in \sigma_{d}(A)} \Phi(d(\lambda)) \leq C\left(\sigma\left(A_{0}\right), \Psi, \Phi, q,\|B\|\right)\|B\|_{\mathcal{S}_{q}}^{q}<\infty .
$$

Example 1. Let $A_{0}$ be a bounded linear operator with a real spectrum, $\sigma\left(A_{0}\right) \subset \mathbb{R}$, and such that condition (5.3) holds with $\Psi(x)=x^{-p}, p>0$. Now both $\sigma_{\text {ess }}\left(A_{0}\right)$ and $\sigma\left(A_{0}\right)$ are compact subsets of the real line, so they are $r$-convex and do not split the plane. So for $A=A_{0}+B, B \in \mathcal{S}_{q}$, and each $\varepsilon>0$, the bounds

$$
\sum_{\lambda \in \sigma_{d}(A)} \Phi(d(\lambda)) \leq C\left(\sigma\left(A_{0}\right), p, q, \varepsilon\right)\|B\|_{\mathcal{S}_{q}}^{q}, \quad \Phi(x)= \begin{cases}x^{p q+1+\varepsilon}, & x \leq 1 \\ x^{p q-\varepsilon}, & x>1\end{cases}
$$

and

$$
\sum_{\lambda \in \sigma_{d}(A)} d^{p q+1+\varepsilon}(\lambda)<\infty
$$

hold. In particular, if $W$ is a bounded linear operator with imaginary component from $\mathcal{S}_{q}$, relations $(5.8)$ and (5.9) are true with $p=1$ and

$$
A_{0}=W_{R}=\frac{W+W^{*}}{2}, \quad B=W_{I}=\frac{W-W^{*}}{2 i} .
$$

A stronger result for self-adjoint $A_{0}$ is in [20]. Its direct application to operators $A_{0}$ similar to a self-adjoint operator, that is, $A_{0}=T^{-1} A_{1} T, A_{1}=A_{1}^{*}$, would lead to a constant $C$ on the right-hand side and depending on the transform $T$. In (5.8) this constant depends only on the spectrum of $A_{0}$.

Example 2. The same argument works for unitary (or similar to unitary) operators $A_{0}$ such that there is $\zeta \in \mathbb{T} \cap \rho\left(A_{0}\right)$. In particular, let $V$ be an $\mathcal{S}_{q}$-quasiunitary operator, that is, $I-V^{*} V \in \mathcal{S}_{q}$, and $\zeta \in \mathbb{T} \cap \rho(V)$. Then its Cayley transform $W=i(\zeta+V)(\zeta-V)^{-1}$ satisfies

$$
W_{I}=\left(\bar{\zeta}-V^{*}\right)^{-1}\left\{I-V^{*} V\right\}(\zeta-V)^{-1} \in \mathcal{S}_{q}
$$

Note that $W+i=2 i \zeta(\zeta-A)^{-1}$ is invertible, and $V=\zeta(W+i)^{-1}(W-i)$. It is easy to see that

$$
V=U+B, \quad B \in \mathcal{S}_{q}, \quad U=\zeta\left(W_{R}+i\right)^{-1}\left(W_{R}-i\right)
$$

is a unitary operator with $\sigma(U) \neq \mathbb{T}$, so the bound similar to (5.9) holds with $A_{0}=U$ and $A=V$. 
Example 3. In the Hilbert space $L^{2}[0,1]$ consider an operator

$$
[A f](x)=a_{0}(x) f(x)+\int_{0}^{1} K(x, y) f(y) d y
$$

with the Hilbert-Schmidt kernel $K$, i.e., $K \in L^{2}([0,1] \times[0,1])$. We assume that the function $a_{0}$ is complex valued, continuous on $[0,1]$, and the arc $\gamma=\left\{a_{0}(x)\right.$ : $0 \leq x \leq 1\}$ is Jordan and either a BC-arc (see Definition 2.7) or has finite global curvature (in particular, $C^{2}$-smooth). The multiplication operator $A_{0} f=a_{0} f$ is normal, and its spectrum $\sigma\left(A_{0}\right)=\gamma$ is the $r$-convex compact set with connected complement (see Propositions 2.4 and 2.8). As in (5.8) we have

$$
\sum_{\lambda \in \sigma_{d}(A)} \Phi(d(\lambda, \gamma)) \leq C(\gamma, \varepsilon)\|K\|_{\mathcal{S}_{2}}^{2}, \quad \Phi(x)= \begin{cases}x^{3+\varepsilon}, & x \leq 1 \\ x^{2-\varepsilon}, & x>1\end{cases}
$$

Acknowledgement. We thank A. Eremenko for helpful remarks concerning $r$-convexity.

\section{References}

[1] Adolfsson, V.: $L^{2}$-integrability of second order derivatives for Poisson's equation in nonsmooth domains. Math. Scand. 70 (1992), 146-160.

[2] Bhatia, R. And Davis, C.: Perturbation of extended enumeration of eigenvalues. Acta Sci. Math. (Szeged) 65 (1999), 277-286.

[3] Blaschke, W.: Eine Erweiterung des Satzes von Vitali über Folgen analytischer Funktionen. S.-B. Sächs Akad. Wiss. Leipzig Math-Natur. Kl. 67 (1915), 194-200.

[4] Borichev, A., Golinskit, L. And Kupin, S.: A Blaschke-type condition and its application to complex Jacobi matrices. Bull. Lond. Math. Soc. 41 (2009), 117-123.

[5] Bouldin, R.: Best approximation of a normal operator in the Schatten $p$-norm. Proc. Amer. Math. Soc. 80 (1980), 277-282.

[6] Carleson, L.: Estimates of harmonic measures. Ann. Acad. Sci. Fenn. Ser. A I Math. 7 (1982), 25-32.

[7] Danzer, L., Grünbaum, B. And Klee, V.: Helly's theorem and its relatives. In Proc. Symp. Pure Math., vol. 7, 101-180. Amer. Math. Soc., Providence, 1963.

[8] Demuth, M., Hansmann, M. and Katriel, G.: On the discrete spectrum of non-selfadjoint operators. J. Funct. Anal. 257 (2009), no. 9, 2742-2759.

[9] DžrbažJAn, M. M.: Theory of factorization and boundary properties of functions meromorphic in the disk. In Proceedings of the International Congress of Mathematicians (Vancouver, B.C., 1974), vol. 2, 197-202. Canad. Math. Congress, Montreal, Que., 1975.

[10] Dunford, N. And Schwartz, J. T.: Linear operators. Part II: Spectral theory. Self-adjoint operators in Hilbert space. John Wiley and Sons, New York-London, 1963.

[11] Dunford, N. And Schwartz, J. T.: Linear operators. Part III: Spectral operators. John Wiley and Sons, New York-London, 1971. 
[12] Favorov, S. And Golinskit, L.: A Blaschke-type condition for analytic and subharmonic functions and application to contraction operators. In Linear and complex analysis, 37-47. Amer. Math Soc. Transl. Ser. 2, 226, Amer. Math. Soc., Providence, RI, 2009.

[13] Favorov, S. and Golinskil, L.: Blaschke-type conditions for analytic and subharmonic functions in the unit disk: local analogs and inverse problems. Comput. Methods Funct. Theory 12 (2012), 151-166.

[14] Garnett, G. B. and Marshall, D.: Harmonic measure. New Mathematical Monographs 2, Cambridge University Press, Cambridge, 2005.

[15] GiL', M. I.: Operator functions and localization of spectra. Lecture Notes in Mathematics 1830, Springer-Verlag, Berlin, 2003.

[16] Gohberg, I. And Krein, M.: Introduction to the theory of linear nonselfadjoint operators. Translations of Mathematical Monographs 18, American Mathematical Society, Providence, RI, 1969.

[17] Golinskit, L. And Kupin, S.: A Blaschke-type condition for analytic functions on finitely connected domains. Applications to complex perturbations of a finite-band selfadjoint operator. J. Math. Anal. Appl. 389 (2012), 705-712.

[18] Golubev, V. V.: The study on the theory of singular points of single valued analytic functions. In Single-valued analytic functions. Automorphic functions. Fiz. Mat. Lit., Moscow, 1961.

[19] Hansmann, M.: An eigenvalue estimate and its application to non-selfadjoint Jacobi and Schrödinger operators. Lett. Math. Phys. 98 (2011), 79-95.

[20] Hansmann, M.: Variation of discrete spectra for non-selfadjoint perturbations of selfadjoint operators. Integral Equations Operator Theory 76 (2013), no. 2, 163-178.

[21] Hansmann, M. and Katriel, G.: Inequalities for the eigenvalues of non-selfadjoint Jacobi operators. Complex Anal. Oper. Theory 5 (2011), 197-218.

[22] Hayman, W. K. and Korenblum, B.: A critical growth rate for functions regular in a disk. Michigan Math. J. 27 (1980), 21-30.

[23] Kato, T.: Variation of discrete spectra. Comm. Math. Phys. 111 (1987), 501-504.

[24] Kato, T.: Perturbation theory for linear operators. Die Grundlehren der mathematischen Wissenschaften 132, Springer-Verlag New York, New York, 1966.

[25] Levin, B. YA.: Lectures on entire functions. Translations of Mathematical Monographs 150, American Mathematical Society, Providence, RI, 1996.

[26] Lieb, E. And Loss, M.: Analysis. Graduate Studies in Mathematics 14, American Mathematical Society, Providence, RI, 1997.

[27] Melnikov, M.: Analytic capacity: a discrete approach and the curvature of measure. Sb. Math. 186 (1995), 827-846.

[28] Milne-Thomson, L. M.: The Calculus of finite differences. Macmillan and Co., London, 1933.

[29] Nevanlinna, R.: Eindeutige analytische Funktionen. (German) Zweite Auflage. Reprint. Die Grundlehren der mathematischen Wissenschaften 46, Springer-Verlag, Berlin-New York, 1974.

[30] Nikolski, N.: Invariant subspaces in operator theory and function theory. J. Soviet Math. 5 (1976), 129-249.

[31] Prasolov, V.: Problems in plane geometry. Moscow textbooks, 2006. 
[32] Putnam, C. R.: Commutation properties of Hilbert space operators and related topics. Ergebnisse der Mathematik und ihrer Grenzgebiete 36, Springer-Verlag New York, New Yoork, 1967.

[33] Ransford, T.: Potential theory in the complex plane. London Mathematical Society Student Texts 28, Cambridge University Press, Cambridge, 1995.

[34] Schuricht, F. And von Der Mosel, H.: Global curvature for rectifiable loops. Math. Z. 243 (2003), 37-77.

[35] Shamoyan, F. A.: Zeros of functions analytic in a disk, that increase near the boundary. Soviet J. Contemporary Math. Anal. 18 (1983), no. 1, 13-25.

[36] Simon, B.: Trace ideals and their applications. Mathematical Surveys and Monographs 120, American Mathematical Society, Providence, RI, 2005.

[37] Singer, I.: Abstract convex analysis. Canadian Mathematical Society Series of Monographs and Advanced Texts, John Wiley \& Sons, New York, 1997.

[38] Stoll, M.: A characterization of Hardy-Orlicz spaces on planar domains. Proc. Amer. Math. Soc. 117 (1993), 1031-1038.

[39] Yulmukhametov, S.: Space of analytic functions with prescribed growth near the boundary. Math. Notes 32 (1982), no. 1-2, 499-508.

Received November 21, 2012; revised April 1, 2013.

Sergey FAvorov: Mathematical School, Karazin Kharkov National University, 4 Swobody sq., 61077 Kharkov, Ukraine.

E-mail: sfavorov@gmail.com

LeOnid Golinskir: Mathematics Division, Institute for Low Temperature Physics and Engineering, 47 Lenin ave., 61103 Kharkov, Ukraine.

E-mail: golinskii@ilt.kharkov.ua 